\title{
Branching Bisimilarity with Explicit Divergence
}

\author{
Rob van Glabbeek \\ National ICT Australia, Sydney, Australia \\ School of Computer Science and Engineering, University of New South Wales, Sydney, Australia
}

Bas Luttik

Department of Mathematics and Computer Science, Technische Universiteit Eindhoven, The Netherlands CWI, The Netherlands

Nikola Trčka

Department of Mathematics and Computer Science, Technische Universiteit Eindhoven, The Netherlands

\begin{abstract}
We consider the relational characterisation of branching bisimilarity with explicit divergence. We prove that it is an equivalence and that it coincides with the original definition of branching bisimilarity with explicit divergence in terms of coloured traces. We also establish a correspondence with several variants of an action-based modal logic with until- and divergence modalities.
\end{abstract}

\section{Introduction}

Branching bisimilarity was proposed in [6]. It is a behavioural equivalence on processes that is compatible with a notion of abstraction from internal activity, while at the same preserving the branching structure of processes in a strong sense. We refer the reader to [6], in particular to Section 10 therein, for ample motivation of the relevance of branching bisimilarity.

Branching bisimilarity abstracts to a large extent from divergence (i.e., infinite internal activity). For instance, it identifies a process, say $P$, that may perform some internal activity after which it returns to its initial state (i.e., $P$ has a $\tau$-loop) with a process, say $P^{\prime}$, that admits the same behaviour as $P$ except that it cannot perform the internal activity leading to the initial state (i.e., $P^{\prime}$ is $P$ without the $\tau$-loop). This means that branching bisimilarity is not compatible with any temporal logic featuring an eventually modality: for any desired state that $P^{\prime}$ will eventually reach, the mentioned internal activity of $P$ may be performed continuously, and thus prevent $P$ from reaching this desired state.

The notion of branching bisimilarity with explicit divergence $\left(\mathrm{BB}^{\Delta}\right)$, also proposed in [6], is a suitable refinement of branching bisimilarity that is compatible with the well-known branching-time temporal logic $\mathrm{CTL}^{*}$ without the nexttime operator $X$ (which is known to be incompatible with abstraction from internal activity). In fact, in [5] we have proved that it is the coarsest semantic equivalence on labelled transition systems with silent moves that is a congruence for parallel composition (as found in process algebras like CCS, CSP or ACP) and only equates processes satisfying the same $\mathrm{CTL}_{-X}^{*}$ formulas. It is also the finest equivalence in the linear time - branching time spectrum of [4].

There are several ways to characterise a behavioural equivalence. The original definition of $\mathrm{BB}^{\Delta}$, in terms of coloured traces, stems from [6]. In [4], $\mathrm{BB}^{\Delta}$ is defined in terms of a modal and a relational characterisation, which are claimed to coincide with each other and with the original notion from [6]. 
Of these three definitions of $\mathrm{BB}^{\Delta}$, the relational characterisation from [4] is the most concise one, in the sense that it requires the least amount of auxiliary concepts. Moreover, this definition is most in the style of the standard definitions of other kinds of bisimulation, found elsewhere in the literature. For these reasons, it is tempting to take it as standard definition.

Although it is not hard to establish that the modal characterisation from [4] is correct, in the sense that it defines an equivalence that coincides with $\mathrm{BB}^{\Delta}$ of [6], it is not at all trivial to establish that the same holds for the relational characterisation from [4]. If fact, it is non-trivial that this relation is an equivalence, and that it satisfies the so-called stuttering property. Once these properties have been established, it follows that the notion coincides with $\mathrm{BB}^{\Delta}$ of [6].

In the remainder of this paper, we shall first, in Section 2 briefly recapitulate the relational, colouredtrace, and modal characterisations of branching bisimilarity. Then, in Section 3, we shall discuss the condition proposed in [4] that can be added to the relational characterisation in order to make it divergence sensitive; we shall then also discuss several variants on this condition. In Section 4 we establish that the relational characterisation of $\mathrm{BB}^{\Delta}$ all coincide, that they are equivalences and that they enjoy the stuttering property. In Section 5 we show that the relational characterisations of $\mathrm{BB}^{\Delta}$ coincide with the original definition of $\mathrm{BB}^{\Delta}$ in terms of coloured traces. Finally, in Section 6 , we shall establish agreement between the relational characterisation from [4], the modal characterisation from [4], and an alternative modal characterisation obtained by adding the divergence modality of [4] to the Hennessy-Milner logic with until proposed in [2].

\section{Branching bisimilarity}

We presuppose a set $A$ of actions with a special element $\tau \in A$, and we presuppose a labelled transition system $(S, \rightarrow)$ with labels from $A$, i.e., $S$ is a set of states and $\rightarrow \subseteq S \times A \times S$ is a transition relation on $S$. Let $s, s^{\prime} \in S$ and $a \in A$. We write $s \stackrel{a}{\longrightarrow} s^{\prime}$ for $\left(s, a, s^{\prime}\right) \in \rightarrow$ and we abbreviate the statement ' $s \stackrel{a}{\longrightarrow} s^{\prime}$ or $\left(a=\tau\right.$ and $\left.s=s^{\prime}\right)$ ' by $s \stackrel{(a)}{\longrightarrow} s^{\prime}$. We denote by $\rightarrow^{+}$the transitive closure of the binary relation $\stackrel{\tau}{\longrightarrow}$, and by $\rightarrow$ its reflexive-transitive closure. A path from a state $s$ is an alternating sequence $s_{0}, a_{1}, s_{1}, a_{2}, s_{2}, \ldots, a_{n}, s_{n}$ of states and actions, such that $s=s_{0}$ and $s_{k-1} \stackrel{a_{k}}{\longrightarrow} s_{k}$ for $k=1, \ldots, n$. A process is given by a state $s$ in a labelled transition system, and encompasses all the states and transitions reachable from $s$.

Relational characterisation The definition of branching bisimilarity that is most widely used has a co-inductive flavour. It defines when a binary relation on states preserves the behaviour of the associated processes. It then declares two states to be equivalent if there exists such a relation relating them. We shall refer to this kind of characterisation as a relational characterisation of branching bisimilarity.

Definition 2.1. A symmetric binary relation $\mathcal{R}$ on $S$ is a branching bisimulation if it satisfies the following condition for all $s, t \in S$ and $a \in A$ :

(T) if $s \mathcal{R} t$ and $s \stackrel{a}{\longrightarrow} s^{\prime}$ for some state $s^{\prime}$, then there exist states $t^{\prime}$ and $t^{\prime \prime}$ such that $t \longrightarrow t^{\prime \prime} \stackrel{(a)}{\longrightarrow} t^{\prime}$, $s \mathcal{R} t^{\prime \prime}$ and $s^{\prime} \mathcal{R} t^{\prime}$

We write $s \unlhd_{b} t$ if there exists a branching bisimulation $\mathcal{R}$ such that $s \mathcal{R} t$. The relation $\unlhd_{b}$ on states is referred to as (the relational characterisation of) branching bisimilarity. 
The relational characterisation of branching bisimilarity presented above is from [4]. As shown in [1, 4, [6], it yields the same concept of branching bisimilarity as the original definition in [6]. The technical advantage of the above definition over the original definition is that the defined notion of branching bisimulation is compositional: the composition of two branching bisimulations is again a branching bisimulation. Basten [1] gives an example showing that the condition used in the original definition of

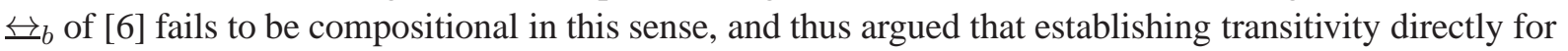
the original definition is not straightforward.

Coloured-trace characterisation To substantiate their claim that branching bisimilarity indeed preserves the branching structure of processes, van Glabbeek and Weijland present in [6] an alternative characterisation of the notion in terms of coloured traces. Below we repeat this characterisation.

Definition 2.2. A colouring is an equivalence on $S$. Given a colouring $\mathcal{C}$ and a state $s \in S$, the colour $\mathcal{C}(s)$ of $s$ is the equivalence class containing $s$.

For $\pi=s_{0}, a_{1}, s_{1}, \ldots, a_{n}, s_{n}$ a path from $s$, let $\mathcal{C}(\pi)$ be the alternating sequence of colours and actions obtained from $\mathcal{C}\left(s_{0}\right), a_{1}, \mathcal{C}\left(s_{1}\right), \ldots, a_{n}, \mathcal{C}\left(s_{n}\right)$ by contracting all subsequences $C, \tau, C, \tau, \ldots, \tau, C$ to $C$. The sequence $\mathcal{C}(\pi)$ is called a $\mathcal{C}$-coloured trace of $s$. A colouring $\mathcal{C}$ is consistent if two states of the same colour always have the same $\mathcal{C}$-coloured traces.

We write $s \equiv_{c} t$ if there exists a consistent colouring $\mathcal{C}$ with $\mathcal{C}(s)=\mathcal{C}(t)$.

In [6] it is proved that $\equiv_{c}$ coincides with the relational characterisation $\bigsqcup_{b}$ of branching bisimilarity.

Modal characterisation A modal characterisation of a behavioural equivalence is a modal logic such that two processes are equivalent iff they satisfy the same formulas of the logic. The modal logic thus corresponding to a behavioural equivalence then allows one, for any two inequivalent processes, to formally express a behavioural property that distinguishes them. Whereas colourings or bisimulations are good tools to show that two processes are equivalent, modal formulas are better for proving inequivalence. The first modal characterisation of a behavioural equivalence is due to Hennessy and Milner [7]. They provided a modal characterisation of (strong) bisimilarity on image-finite labelled transition systems, using a modal logic that is nowadays referred to as the Hennessy-Milner Logic. The modal characterisations of branching bisimilarity presented below are adaptations of the Hennessy-Milner Logic.

The class of formulas $\Phi_{j b}$ of the modal logic for branching bisimilarity proposed in [4] is generated by the following grammar:

$$
\varphi::=\neg \varphi|\bigwedge \Phi| \varphi a \varphi \quad\left(a \in A, \varphi \in \Phi_{j b} \text { and } \Phi \subseteq \Phi_{j b}\right) .
$$

In case the cardinality $|S|$ of the set of states of our labelled transition system is less than some infinite cardinal $\kappa$, we may require that $|\Phi|<\kappa$ in conjunctions, thus obtaining a set of formulas rather than a proper class. We shall use the following standard abbreviations: $\top=\bigwedge \emptyset, \perp=\neg \top$ and $\bigvee \Phi=$ $\neg \bigwedge\{\neg \varphi \mid \varphi \in \Phi\}$.

We define when a formula $\varphi$ is valid in a state $s$ (notation: $s \models \varphi$ ) inductively as follows:

(i) $s \models \neg \varphi$ iff $s \not \models \varphi$;

(ii) $s \models \bigwedge \Phi$ iff $s \models \varphi$ for all $\varphi \in \Phi$; 
(iii) $s \models \varphi a \psi$ iff there exist states $s^{\prime}$ and $s^{\prime \prime}$ such that $s \longrightarrow s^{\prime \prime} \stackrel{(a)}{\longrightarrow} s^{\prime}, s^{\prime \prime} \models \varphi$ and $s^{\prime} \models \psi$.

Validity induces an equivalence on states: we define $\approx \subseteq S \times S$ by

$$
s \approx t \quad \text { iff } \quad \forall \varphi \in \Phi_{j b} . s \models \varphi \Leftrightarrow t \models \varphi .
$$

In [4] it was shown that $\approx$ coincides with $\unlhd_{b}$, that is, branching bisimilarity is characterised by the modal logic above.

Clause (iii) in the definition of validity appears to be rather liberal. More stringent alternatives are obtained by using $\varphi\langle\hat{a}\rangle \psi$ or $\varphi\langle a\rangle \psi$ instead of $\varphi a \psi$, with the following definitions:

(iii' $^{\prime} s \models \varphi\langle\hat{a}\rangle \psi$ iff either $a=\tau$ and $s \models \psi$, or there exists a sequence of states $s_{0}, \ldots, s_{n}, s_{n+1}$ $(n \geq 0)$ such that $s=s_{0} \stackrel{\tau}{\longrightarrow} \cdots \stackrel{\tau}{\longrightarrow} s_{n} \stackrel{a}{\longrightarrow} s_{n+1}, s_{i} \models \varphi$ for all $i=0, \ldots, n$ and $s_{n+1} \models \psi$.

(iii") $s \models \varphi\langle a\rangle \psi$ iff there exists states $s_{0}, \ldots, s_{n}, s_{n+1}(n \geq 0)$ such that $s=s_{0} \stackrel{\tau}{\longrightarrow} \ldots \stackrel{\tau}{\longrightarrow} s_{n} \stackrel{(a)}{\longrightarrow} s_{n+1}$, $s_{i} \models \varphi$ for all $i=0, \ldots, n$ and $s_{n+1} \models \psi$.

The modality $\langle\hat{a}\rangle$ stems from De Nicola \& Vaandrager [2]. There it was shown, for labelled transition systems with bounded nondeterminism, that branching bisimilarity, $\unlhd_{b}$, is characterised by the logic with negation, binary conjunction and this until modality. The modality $\langle a\rangle$ is a common strengthening of $\langle\hat{a}\rangle$ and the just-before modality $a$ above; it was first considered in [4].

To be able to compare the expressiveness of modal logics, the following definitions are proposed by Laroussinie, Pinchinat \& Schnoebelen [8].

Definition 2.3. Two modal formulas $\varphi$ and $\psi$ that are interpreted on states of labelled transition systems are equivalent, written $\varphi \Leftrightarrow \psi$, if $s \models \varphi \Leftrightarrow s \models \psi$ for all states $s$ in all labelled transition systems. Two modal logics are equally expressive if for every formula in the one there is an equivalent formula in the other.

As remarked in [4], the modalities $\langle\hat{a}\rangle$ and $\langle a\rangle$ are equally expressive, since

$$
\begin{aligned}
\varphi\langle\hat{\tau}\rangle \psi & \Leftrightarrow \psi \vee \varphi\langle\tau\rangle \psi, \\
\varphi\langle\tau\rangle \psi & \Leftrightarrow \varphi \wedge \varphi\langle\hat{\tau}\rangle \psi \text { and } \\
\varphi\langle a\rangle \psi & \Leftrightarrow \varphi\langle\hat{a}\rangle \psi \quad \text { for all } a \neq \tau .
\end{aligned}
$$

Note that the modality $a$ can be expressed in terms of $\langle a\rangle$ :

$$
\varphi a \psi \Leftrightarrow \top\langle\tau\rangle(\varphi\langle a\rangle \psi)
$$

Laroussinie, Pinchinat \& Schnoebelen established in [8] that the modal logic with negation, binary conjunction and $a$ from [4] and the logic with negation, binary conjunction and $\langle\hat{a}\rangle$ from [2] are equally expressive. 


\section{Relational characterisations of $\mathbf{B B}^{\Delta}$}

The notion branching bisimilarity discussed in the previous section abstracts from divergence (i.e, infinite internal activity). In the remainder of this paper, we discuss a refinement of the notion of branching bisimulation equivalence that takes divergence into account. In this section we present several conditions that can be added to the notion of branching bisimulation in order to make it divergence sensitive. The induced notions of branching bisimilarity with explicit divergence will all turn out to be equivalent.

Definition 3.1. [4] A symmetric binary relation $\mathcal{R}$ on $S$ is a branching bisimulation with explicit divergence if it is a branching bisimulation (i.e., it satisfies condition (T) of Definition 2.1) and in addition satisfies the following condition for all $s, t \in S$ and $a \in A$ :

(D) if $s \mathcal{R} t$ and there is an infinite sequence of states $\left(s_{k}\right)_{k \in \omega}$ such that $s=s_{0}, s_{k} \stackrel{\tau}{\longrightarrow} s_{k+1}$ and $s_{k} \mathcal{R} t$ for all $k \in \omega$, then there exists an infinite sequence of states $\left(t_{\ell}\right)_{\ell \in \omega}$ such that $t=t_{0}$, $t_{\ell} \stackrel{\tau}{\longrightarrow} t_{\ell+1}$ for all $\ell \in \omega$, and $s_{k} \mathcal{R} t_{\ell}$ for all $k, \ell \in \omega$.

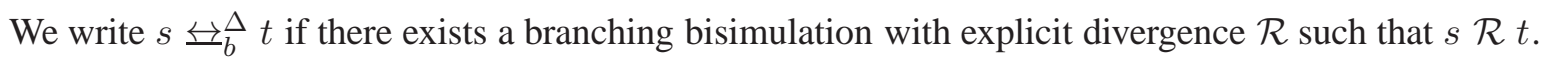

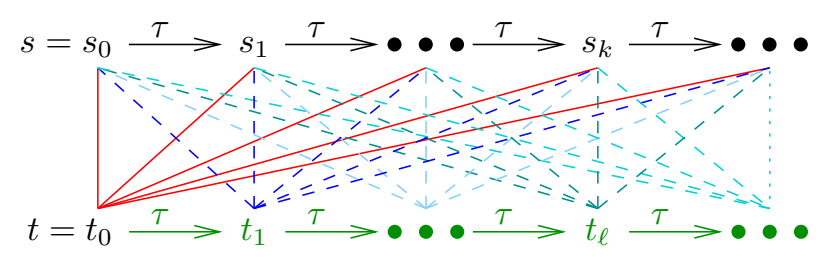

Figure 1. Condition (D).

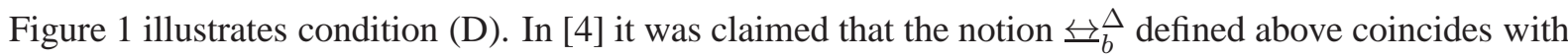
branching bisimilarity with explicit divergence as defined earlier in [6]. In this paper we will substantiate

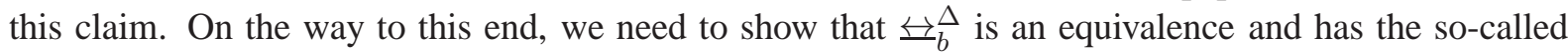
stuttering property.

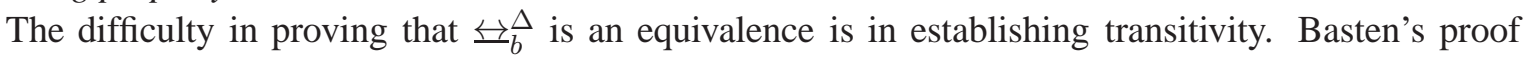
in [1] that $\unlhd_{b}$ (i.e., branching bisimilarity without explicit divergence) is transitive, is obtained as an immediate consequence of the fact that whenever two binary relations $\mathcal{R}_{1}$ and $\mathcal{R}_{2}$ satisfy (T), then so does their composition $\mathcal{R}_{1} ; \mathcal{R}_{2}$ (see Lemma 4.3 below). The condition (D) fails to be compositional, as we show in the following example.

Example 3.1. Consider the labelled transition system depicted on the left-hand side of Figure 2 together with the branching bisimulations with explicit divergence

$$
\begin{aligned}
& \mathcal{R}_{1}=\left\{\left(s_{0}, t_{0}\right),\left(t_{0}, s_{0}\right),\left(s_{1}, t_{1}\right),\left(t_{1}, s_{1}\right),\left(s_{2}, t_{2}\right),\left(t_{2}, s_{2}\right),\left(s_{1}, t_{2}\right),\left(t_{2}, s_{1}\right),\left(s_{2}, t_{1}\right),\left(t_{1}, s_{2}\right)\right\} \quad \text { and } \\
& \mathcal{R}_{2}=\left\{\left(t_{0}, u_{0}\right),\left(u_{0}, t_{0}\right),\left(t_{1}, u_{1}\right),\left(u_{1}, t_{1}\right),\left(t_{2}, u_{2}\right),\left(u_{2}, t_{2}\right),\left(t_{0}, u_{1}\right),\left(u_{1}, t_{0}\right),\left(t_{1}, u_{0}\right),\left(u_{0}, t_{1}\right)\right\} .
\end{aligned}
$$

The composition $\mathcal{R}=\mathcal{R}_{1} ; \mathcal{R}_{2}$ on the relevant fragment is depicted on the right-hand side of Figure 2 Note that $s_{0}$ gives rise to a divergence of which every state is related by $\mathcal{R}$ to $u_{0}$. However, since $s_{0}$ and 

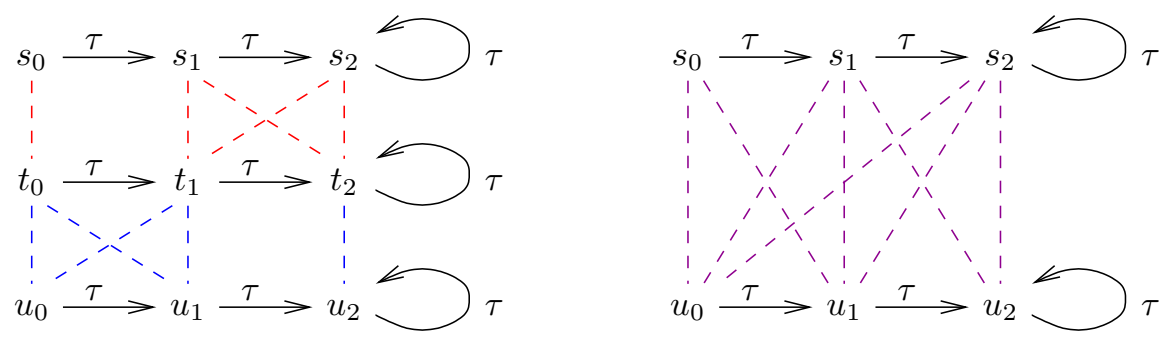

Figure 2. The composition of branching bisimulations with explicit divergence is not a branching bisimulation with explicit divergence.

$u_{2}$ are not related according to $\mathcal{R}$, there is no divergence from $u_{0}$ of which every state is related to every state on the divergence from $s_{0}$. We conclude that $\mathcal{R}$ does not satisfy the condition (D).

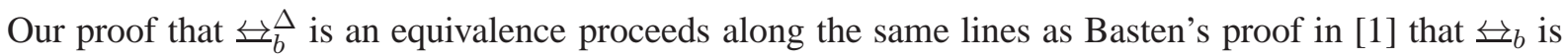
an equivalence: we replace $(\mathrm{D})$ by an alternative divergence condition that is compositional, prove that the resulting notion of bisimilarity is an equivalence, and then establish that it coincides with $\unlhd_{b}^{\Delta}$. In the remainder of this section, we shall arrive at our compositional alternative for (D) through a series of adaptations of (D).

First, we observe that (D) has a technically convenient reformulation: instead of requiring the existence of a divergence from $t$ all the states of which enjoy certain properties, it suffices to require that there exists a state reachable from $t$ by a single $\tau$-transition with these properties. Formally, the reformulation of (D) is:

$\left(\mathrm{D}_{0}\right)$ if $s \mathcal{R} t$ and there is an infinite sequence of states $\left(s_{k}\right)_{k \in \omega}$ such that $s=s_{0}, s_{k} \stackrel{\tau}{\longrightarrow} s_{k+1}$ and $s_{k} \mathcal{R} t$ for all $k \in \omega$, then there exists a state $t^{\prime}$ such that $t \stackrel{\tau}{\longrightarrow} t^{\prime}$ and $s_{k} \mathcal{R} t^{\prime}$ for all $k \in \omega$.

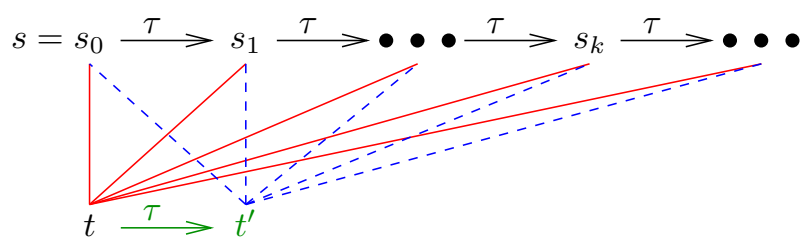

Figure 3. Condition ( $\mathrm{D}_{0}$.

Figure 3 illustrates condition $\left(\mathrm{D}_{0}\right)$. If a binary relation satisfies $\left(\mathrm{D}_{0}\right)$, then the divergence from $t$ required by $(\mathrm{D})$ can be inductively constructed. (We omit the inductive construction here; the proof of Proposition 3.1 below contains a very similar inductive construction.)

For our next adaptation we observe that $\left(\mathrm{D}_{0}\right)$ has some redundancy. Note that it requires $t^{\prime}$ to be related to every state on the divergence from $s$. However, the universal quantification in the conclusion can be relaxed to an existential quantification: it suffices to require that $t$ has an immediate $\tau$-successor that is related to some state on the divergence from $s$. The requirement can be expressed as follows: 
$\left(\mathrm{D}_{1}\right)$ if $s \mathcal{R} t$ and there is an infinite sequence of states $\left(s_{k}\right)_{k \in \omega}$ such that $s=s_{0}, s_{k} \stackrel{\tau}{\longrightarrow} s_{k+1}$ and $s_{k} \mathcal{R} t$ for all $k \in \omega$, then there exists a state $t^{\prime}$ such that $t \stackrel{\tau}{\longrightarrow} t^{\prime}$ and $s_{k} \mathcal{R} t^{\prime}$ for some $k \in \omega$.

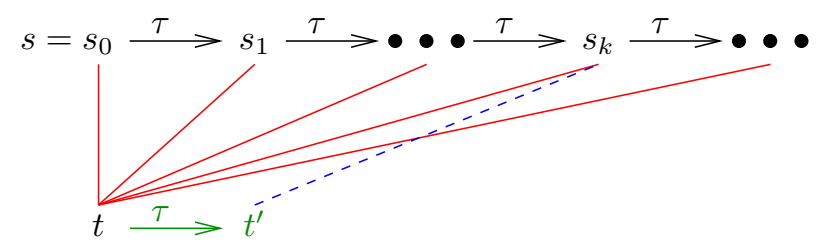

Figure 4. Condition $\left(\mathrm{D}_{1}\right)$.

Condition $\left(\mathrm{D}_{1}\right)$ appears in the definition of divergence-sensitive stuttering simulation of Nejati [9]. It is

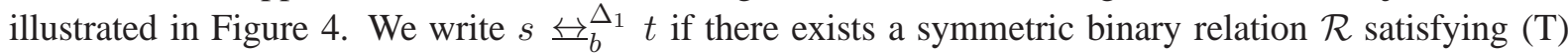
and $\left(\overline{\mathrm{D}_{1}}\right)$ such that $s \mathcal{R} t$. Note that every relation satisfying $(\mathrm{D})$ also satisfies $\left(\overline{\mathrm{D}_{1}}\right)$, so it follows that

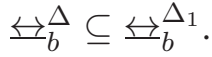

The following example illustrates that condition $\left(\overline{\mathrm{D}_{1}}\right)$ is still not compositional, not even if the composed relations satisfy (T).
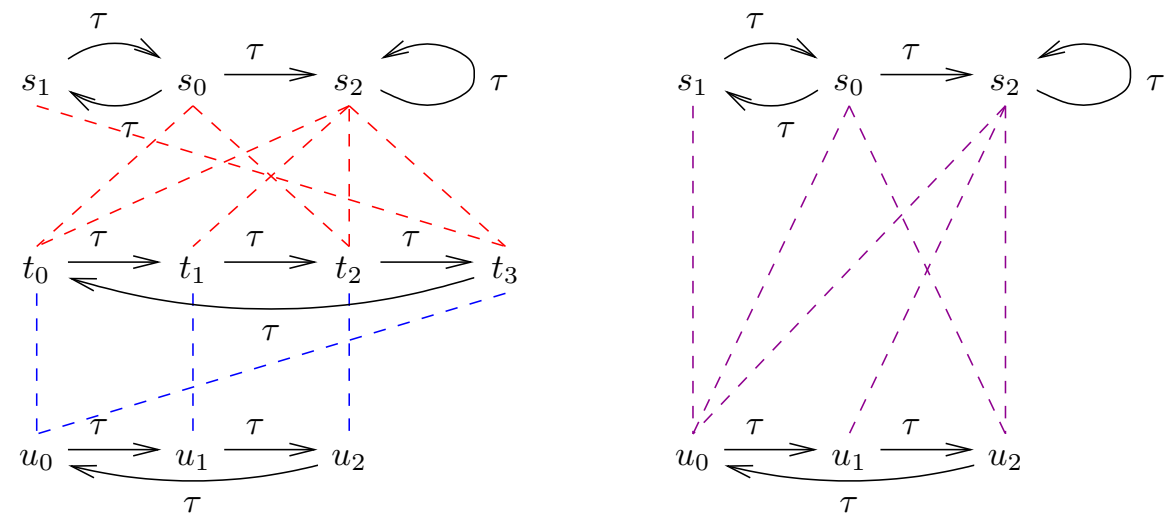

Figure 5. The composition of binary relations satisfying $(T)$ and $\left(\mathrm{D}_{1}\right)$ does not necessarily satisfy $\left(\mathrm{D}_{1}\right)$.

Example 3.2. Consider the labelled transition system depicted on the left-hand side of Figure 5 together with two binary relations satisfying $(\mathrm{T})$ and $\left(\overline{\mathrm{D}_{1}}\right)$ :

$$
\begin{aligned}
& \mathcal{R}_{1}=\left\{\left(s_{0}, t_{0}\right),\left(t_{0}, s_{0}\right),\left(s_{0}, t_{2}\right),\left(t_{2}, s_{0}\right),\left(s_{1}, t_{3}\right),\left(t_{3}, s_{1}\right)\right\} \cup\left\{\left(s_{2}, t_{i}\right),\left(t_{i}, s_{2}\right) \mid 0 \leq i \leq 3\right\} \text { and } \\
& \mathcal{R}_{2}=\left\{\left(t_{i}, u_{i}\right),\left(u_{i}, t_{i}\right) \mid 0 \leq i \leq 2\right\} \cup\left\{\left(t_{3}, u_{0}\right),\left(u_{0}, t_{3}\right)\right\} .
\end{aligned}
$$

Note that, since $s_{1}$ is not $\mathcal{R}_{1}$-related to $t_{0}$, the divergence $s_{0} \stackrel{\tau}{\longrightarrow} s_{1} \stackrel{\tau}{\longrightarrow} s_{0} \stackrel{\tau}{\longrightarrow} s_{1} \stackrel{\tau}{\longrightarrow} \cdots$ need not be simulated by $t_{0}$ in such a way that $t_{1}$ is related to either $s_{0}$ or $s_{1}$.

Now consider the composition $\mathcal{R}=\mathcal{R}_{1} ; \mathcal{R}_{2}$. Both $s_{0}$ and $s_{1}$ are $\mathcal{R}$-related to $u_{0}$, whereas the state $u_{1}$ is not $\mathcal{R}$-related to $s_{0}$ nor to $s_{1}$. We conclude that $\mathcal{R}$ does not satisfy $\left(\mathrm{D}_{1}\right)$. 
The culprit in the preceding example appears to be the fact that $\left(\mathrm{D}_{1}\right)$ only considers divergences from $s$ of which every state is related to $t$. Our second alternative omits this restriction. It considers every divergence from $s$ and requires that it is simulated by $t$.

$\left(\mathrm{D}_{2}\right)$ if $s \mathcal{R} t$ and there is an infinite sequence of states $\left(s_{k}\right)_{k \in \omega}$ such that $s=s_{0}$ and $s_{k} \stackrel{\tau}{\longrightarrow} s_{k+1}$ for all $k \in \omega$, then there exists a state $t^{\prime}$ such that $t \stackrel{\tau}{\longrightarrow} t^{\prime}$ and $s_{k} \mathcal{R} t^{\prime}$ for some $k \in \omega$.

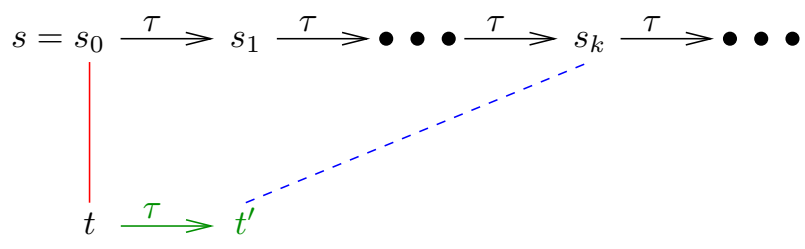

Figure 6. Condition $\left(\mathrm{D}_{2}\right)$.

Figure 6 illustrates condition $\left(\mathrm{D}_{2}\right)$. In contrast to the preceding divergence conditions, it does have the property that if two relations both satisfy it, then so does their relational composition. However, to facilitate a direct proof of this property, it is technically convenient to reformulate condition $\left(\mathrm{D}_{2}\right)$ such that it requires a divergence from $t$ rather than just one $\tau$-step:

$\left(\mathrm{D}_{3}\right)$ if $s \mathcal{R} t$ and there is an infinite sequence of states $\left(s_{k}\right)_{k \in \omega}$ such that $s=s_{0}$ and $s_{k} \stackrel{\tau}{\longrightarrow} s_{k+1}$ for all $k \in \omega$, then there exist an infinite sequence of states $\left(t_{\ell}\right)_{\ell \in \omega}$ and a mapping $\sigma: \omega \rightarrow \omega$ such that $t=t_{0}, t_{\ell} \stackrel{\tau}{\longrightarrow} t_{\ell+1}$ and $s_{\sigma(\ell)} \mathcal{R} t_{\ell}$ for all $\ell \in \omega$.

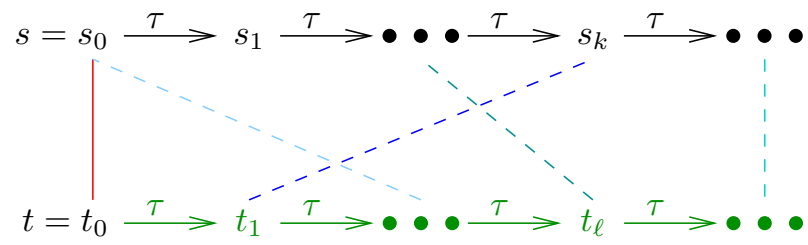

Figure 7. Condition $\left(\mathrm{D}_{3}\right)$.

Figure 7 illustrates condition $\left(\overline{\mathrm{D}_{3}}\right)$.

Proposition 3.1. A binary relation $\mathcal{R}$ satisfies $\left(\overline{D_{2}}\right)$ iff it satisfies $\left(\overline{D_{3}}\right)$.

Proof The implication from right to left is trivial. For the implication from left to right, suppose that $\mathcal{R}$ satisfies $\left(\mathrm{D}_{2}\right)$ and that $s \mathcal{R} t$, and consider an infinite sequence of states $\left(s_{k}\right)_{k \in \omega}$ such that $s=s_{0}$ and $s_{k} \stackrel{\tau}{\longrightarrow} s_{k+1}$ for all $k \in \omega$. We construct an infinite sequence of states $\left(t_{\ell}\right)_{\ell \in \omega}$ and a mapping $\sigma: \omega \rightarrow \omega$ such that $t=t_{0}, t_{\ell} \stackrel{\tau}{\longrightarrow} t_{\ell+1}$ and $s_{\sigma(\ell)} \mathcal{R} t_{\ell}$ for all $\ell \in \omega$.

The infinite sequence $\left(t_{\ell}\right)_{\ell \in \omega}$ and the mapping $\sigma: \omega \rightarrow \omega$ can be defined simultaneously by induction on $l$ : 
1. We define $t_{0}=t$ and $\sigma(0)=0$; it then clearly holds that $s_{\sigma(0)} \mathcal{R} t_{0}$.

2. Suppose that the sequence $\left(t_{\ell}\right)_{\ell \in \omega}$ and the mapping $\sigma: \omega \rightarrow \omega$ have been defined up to $\ell$. Then, in particular, $s_{\sigma(\ell)} \mathcal{R} t_{\ell}$. Since $\left(s_{\sigma(\ell)+k}\right)_{k \in \omega}$ is an infinite sequence such that $s_{\sigma(\ell)+k} \stackrel{\tau}{\longrightarrow} s_{\sigma(\ell)+k+1}$ for all $k \in \omega$, by $\left(\overline{\mathrm{D}_{2}}\right)$ there exists $t^{\prime}$ such that $t_{\ell} \stackrel{\tau}{\longrightarrow} t^{\prime}$ and $s_{\sigma(\ell)+k^{\prime}} \mathcal{R} t^{\prime}$ for some $k^{\prime} \in \omega$. We define $t_{\ell+1}=t^{\prime}$ and $\sigma(\ell+1)=k^{\prime}$.

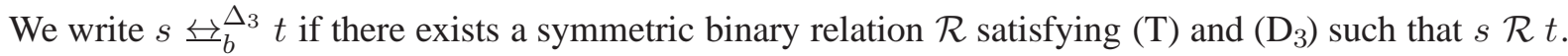
Note that $\left(\overline{D_{1}}\right)$ is a weaker requirement than $\left(\overline{D_{2}}\right)$, and hence, by Proposition 3.1 , than $\left(\mathrm{D}_{3}\right)$. It follows that $\overleftrightarrow{b}_{b}^{\Delta_{3}} \subseteq \overleftrightarrow{B}_{b}^{\Delta_{1}}$. Also note that $\left(\overline{\mathrm{D}_{2}}\right)$ and $\left(\underline{\left.\mathrm{D}_{3}\right)}\right.$ on the one hand and $(\mathrm{D})$ and $\left(\overline{\mathrm{D}_{0}}\right)$ on the other hand are incomparable.

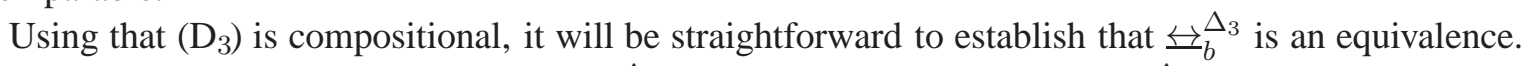

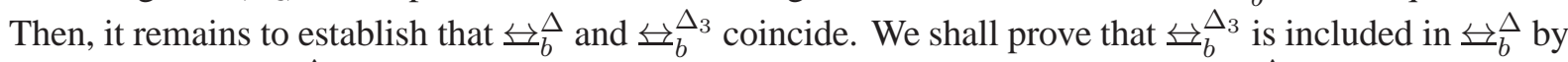

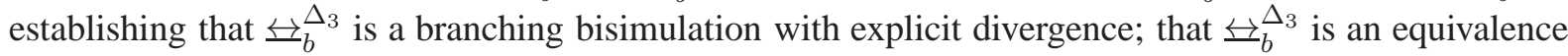
is crucial in the proof of this property. Instead of proving the converse inclusion directly, we obtain a stronger result by establishing that a notion of bisimilarity defined using a weaker divergence condition

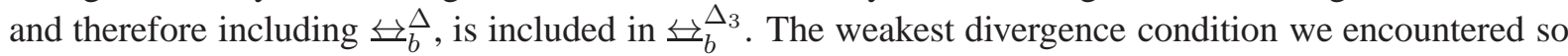
far is $\left(\mathrm{D}_{1}\right)$. It is, however, possible to further weaken $\left(\mathrm{D}_{1}\right)$ : instead of requiring that $t^{\prime}$ is an immediate $\tau$-successor, it is enough require that $t^{\prime}$ can be reached from $t$ by one or more $\tau$-transitions. Formally,

$\left(\mathrm{D}_{4}\right)$ if $s \mathcal{R} t$ and there is an infinite sequence of states $\left(s_{k}\right)_{k \in \omega}$ such that $s=s_{0}, s_{k} \stackrel{\tau}{\longrightarrow} s_{k+1}$ and $s_{k} \mathcal{R} t$ for all $k \in \omega$, then there exists a state $t^{\prime}$ such that $t \longrightarrow^{+} t^{\prime}$ and $s_{k} \mathcal{R} t^{\prime}$ for some $k \in \omega$.

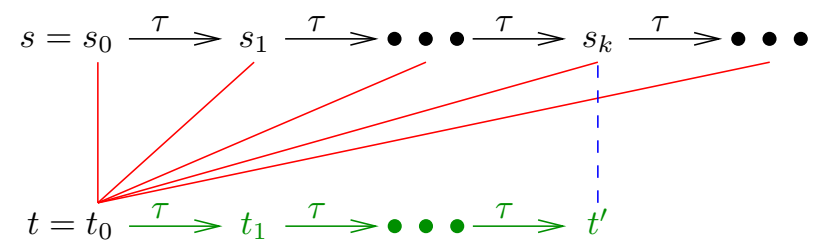

Figure 8. Condition $\left(\mathrm{D}_{4}\right.$.

Figure 8 illustrates condition $\left(\overline{\mathrm{D}_{4}}\right)$. We write $s \overleftrightarrow{\unlhd}_{b}^{\Delta_{4}} t$ if there exists a symmetric binary relation $\mathcal{R}$

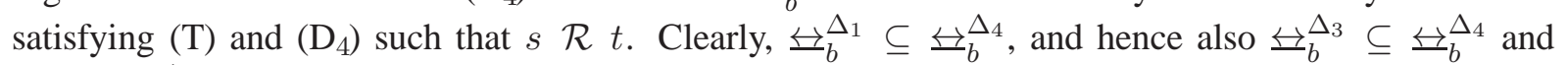

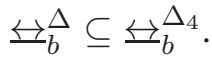

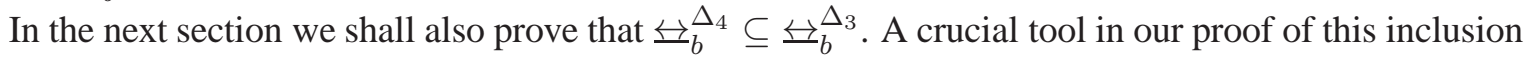
will be the notion of stuttering closure of a binary relation $\mathcal{R}$ on states. The stuttering closure of $\mathcal{R}$ enjoys the so-called stuttering property: if from state $s$ a state $s^{\prime}$ can be reached through a sequence of $\tau$-transitions, and both $s$ and $s^{\prime}$ are $\mathcal{R}$-related to the same state $t$, then all intermediate states between $s$ and $s^{\prime}$ are $\mathcal{R}$-related to $t$ too. We shall prove a lemma to the effect that if a binary relation on states satisfies $(\mathrm{T})$ and $\left(\overline{\mathrm{D}_{4}}\right)$, then its stuttering closure satisfies $(\mathrm{T})$ and $\left(\mathrm{D}_{3}\right)$, and use it to establish the inclusion

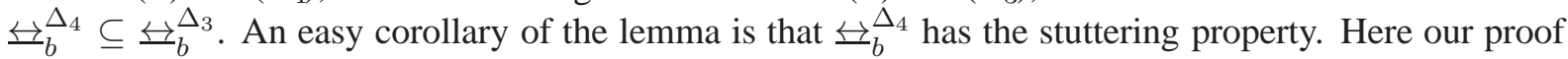
also has a similarity with Basten's proof in [1]; in his proof that the notions of branching bisimilarity 


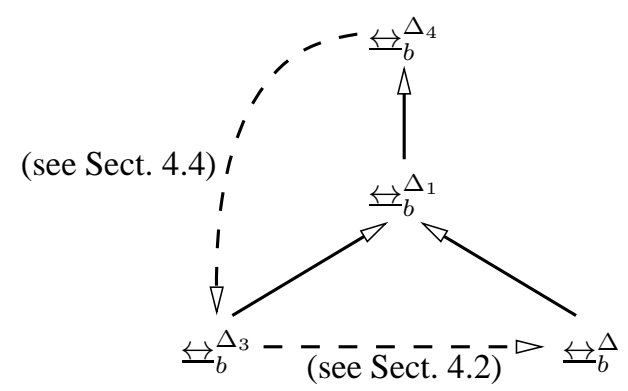

Figure 9. Inclusion graph.

induced by (T) and by the original condition used in [6] coincide, establishing the stuttering property is a crucial step.

Figure 9 shows some inclusions between the different versions of branching bisimilarity with explicit divergence. (Note that we never defined $\unlhd_{b}^{\Delta_{0}}$ and $\unlhd_{b}^{\Delta_{2}}$, as these would be the same as $\unlhd_{b}^{\Delta}$ and $\bigsqcup_{b}^{\Delta_{3}}$, respectively.) The solid arrows indicate inclusions that have already been argued for above; the dashed arrows indicate inclusions that will be established below.

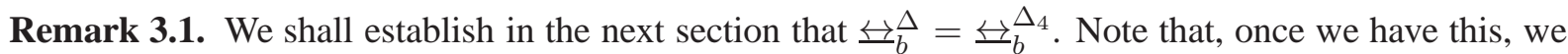
can replace the second condition of Definition 3.1 by any interpolant of $(\mathrm{D})$ and $\left(\mathrm{D}_{4}\right)$, i.e., any condition that is implied by $(\mathrm{D})$ and implies $\left(\mathrm{D}_{4}\right)$, and end up with the same equivalence. For instance, we could replace it by condition $\left(\mathrm{D}_{1}\right)$, or by the condition of Gerth, Kuiper, Peled \& Penczek [3]:

if $s \mathcal{R} t$ and there is an infinite sequence of states $\left(s_{k}\right)_{k \in \omega}$ such that $s=s_{0}, s_{k} \stackrel{\tau}{\longrightarrow} s_{k+1}$ and $s_{k} \mathcal{R} t$ for all $k \in \omega$, then there exists a state $t^{\prime}$ such that $t \stackrel{\tau}{\longrightarrow} t^{\prime}$ and $s_{k} \mathcal{R} t^{\prime}$ for some $k>0$.

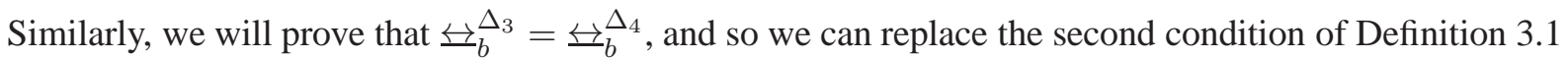
by an interpolant of $\left(\overline{\mathrm{D}_{3}}\right)$ and $\left(\overline{\mathrm{D}_{4}}\right)$. For instance, the condition

if $s \mathcal{R} t$ and there is an infinite sequence of states $\left(s_{k}\right)_{k \in \omega}$ such that $s=s_{0}$ and $s_{k} \stackrel{\tau}{\longrightarrow} s_{k+1}$ for all $k \in \omega$, then there exists a state $t^{\prime}$ such that $t \longrightarrow^{+} t^{\prime}$ and $s_{k} \mathcal{R} t^{\prime}$ for some $k \geq 0$

is a convenient interpolant of $\left(\overline{D_{3}}\right)$ and $\left(\overline{D_{4}}\right)$ to use when showing that two states are branching bisimulation equivalent with explicit divergence.

\section{4. $\mathrm{BB}^{\Delta}$ is an equivalence with the stuttering property}

Our goal is now to establish that the relational characterisations of branching bisimilarity with explicit divergence introduced in the previous section all coincide, that they are equivalences and that they enjoy the stuttering property. To this end, we first show that $\overleftrightarrow{ }_{b}^{\Delta_{3}}$ is an equivalence relation; condition $\left(\mathrm{D}_{3}\right)$ will

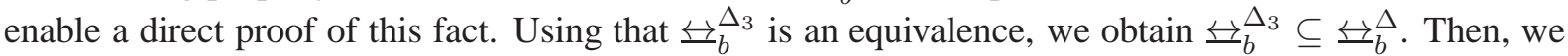

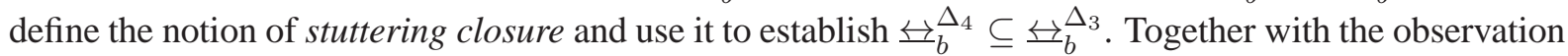

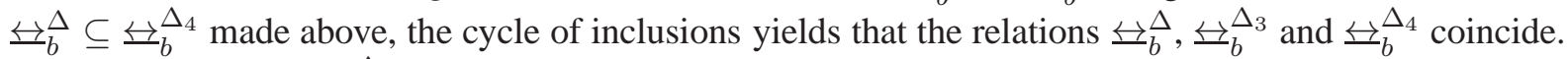
It then follows that $\unlhd_{b}$ is an equivalence. We have not been able to find a less roundabout way to 
obtain this result. The intermediate results needed for the equivalence proof also yields that $\unlhd_{b}^{\Delta}$ has the stuttering property.

\section{1. $\overleftrightarrow{ }_{b}^{\Delta_{3}}$ is an equivalence}

The proofs below are rather straightforward. Nevertheless, the proof strategy employed for Lemmas 4.1

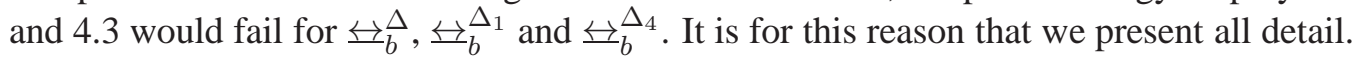

Lemma 4.1. Let $\left\{\mathcal{R}_{i} \mid i \in I\right\}$ be a family of binary relations.

(i) If $\mathcal{R}_{i}$ satisfies (T) for all $i \in I$, then so does the union $\bigcup_{i \in I} \mathcal{R}_{i}$.

(ii) If $\mathcal{R}_{i}$ satisfies $\left(\overline{\mathrm{D}_{3}}\right)$ for all $i \in I$, then so does the union $\bigcup_{i \in I} \mathcal{R}_{i}$.

Proof Let $\mathcal{R}=\bigcup_{i \in I} \mathcal{R}_{i}$.

(i) Suppose that $\mathcal{R}_{i}$ satisfies (T) for all $i \in I$. To prove that $\mathcal{R}$ also satisfies (T), suppose that $s \mathcal{R} t$ and $s \stackrel{a}{\longrightarrow} s^{\prime}$ for some state $s^{\prime}$. Then $s \mathcal{R}_{i} t$ for some $i \in I$. Since $\mathcal{R}_{i}$ satisfies $[T$, it follows that there are states $t^{\prime}$ and $t^{\prime \prime}$ such that $t \longrightarrow t^{\prime \prime} \stackrel{(a)}{\longrightarrow} t^{\prime}, s \mathcal{R}_{i} t^{\prime \prime}$ and $s^{\prime} \mathcal{R}_{i} t^{\prime}$, and hence $s \mathcal{R} t^{\prime \prime}$ and $s^{\prime} \mathcal{R} t^{\prime}$.

(ii) Suppose that $\mathcal{R}_{i}$ satisfies $\left(\mathrm{D}_{3}\right)$ for all $i \in I$. To prove that $\mathcal{R}$ satisfies $\left(\overline{\mathrm{D}_{3}}\right)$, suppose that $s \mathcal{R} t$ and that there is an infinite sequence of states $\left(s_{k}\right)_{k \in \omega}$ such that $s=s_{0}$ and $s_{k} \stackrel{\tau}{\longrightarrow} s_{k+1}$. From $s \mathcal{R} t$ it follows that $s \mathcal{R}_{i} t$ for some $i \in I$. By $\left(\overline{\mathrm{D}_{3}}\right)$ there exist an infinite sequence of states $\left(t_{\ell}\right)_{\ell \in \omega}$ and a mapping $\sigma: \omega \rightarrow \omega$ such that $t=t_{0}, t_{\ell} \stackrel{\tau}{\longrightarrow} t_{\ell+1}$ and $s_{\sigma(\ell)} \mathcal{R}_{i} t_{\ell}$ for all $\ell \in \omega$, and from the latter it follows that $s_{\sigma(\ell)} \mathcal{R} t_{\ell}$ for all $\ell \in \omega$.

Lemma 4.2. Let $\mathcal{R}$ be a binary relation that satisfies $(T)$. If $s \mathcal{R} t$ and $s \longrightarrow s^{\prime}$, then there is a state $t^{\prime}$ such that $t \longrightarrow t^{\prime}$ and $s^{\prime} \mathcal{R} t^{\prime}$.

Proof Let $s_{0}, \ldots, s_{n}$ be states such that $s=s_{0} \stackrel{\tau}{\longrightarrow} \cdots \stackrel{\tau}{\longrightarrow} s_{n}=s^{\prime}$. By (T) and a straightforward induction on $n$ there exist states $t_{0}, \ldots, t_{n}$ such that $t=t_{0} \longrightarrow \cdots \longrightarrow t_{n}=t^{\prime}$ and $s_{i} \mathcal{R} t_{i}$ for all $i \leq n$.

Lemma 4.3. Let $\mathcal{R}_{1}$ and $\mathcal{R}_{2}$ be binary relations.

(i) If $\mathcal{R}_{1}$ and $\mathcal{R}_{2}$ both satisfy (T), then so does their composition $\mathcal{R}_{1} ; \mathcal{R}_{2}$.

(ii) If $\mathcal{R}_{1}$ and $\mathcal{R}_{2}$ both satisfy $\left(\overline{\mathrm{D}}_{3}\right)$, then so does their composition $\mathcal{R}_{1} ; \mathcal{R}_{2}$.

Proof Let $\mathcal{R}=\mathcal{R}_{1} ; \mathcal{R}_{2}$.

(i) To prove that $\mathcal{R}$ satisfies $\left(\mathrm{T}\right.$, suppose $s \mathcal{R} u$ and $s \stackrel{a}{\longrightarrow} s^{\prime}$. Then there exists a state $t$ such that $s \mathcal{R}_{1} t$ and $t \mathcal{R}_{2} u$. Since $\mathcal{R}_{1}$ satisfies $\left(\mathrm{T}\right.$ ), there exist states $t^{\prime}$ and $t^{\prime \prime}$ such that $t \longrightarrow t^{\prime \prime} \stackrel{(a)}{\longrightarrow} t^{\prime}$, $s \mathcal{R}_{1} t^{\prime \prime}$ and $s^{\prime} \mathcal{R}_{1} t^{\prime}$. By Lemma 4.2 there is a state $u^{\prime \prime}$ such that $u \longrightarrow u^{\prime \prime}$ and $t^{\prime \prime} \mathcal{R}_{2} u^{\prime \prime}$. We now distinguish two cases:

(a) Suppose that $a=\tau$ and $t^{\prime \prime}=t^{\prime}$. Then $u \longrightarrow u^{\prime \prime} \stackrel{(a)}{\longrightarrow} u^{\prime \prime}$, from $s \mathcal{R}_{1} t^{\prime \prime}$ and $t^{\prime \prime} \mathcal{R}_{2} u^{\prime \prime}$ it follows that $s \mathcal{R} u^{\prime \prime}$, and from $s^{\prime} \mathcal{R}_{1} t^{\prime}$ and $t^{\prime} \mathcal{R}_{2} u^{\prime \prime}$ it follows that $s^{\prime} \mathcal{R} u^{\prime \prime}$. 
(b) Suppose that $t^{\prime \prime} \stackrel{a}{\longrightarrow} t^{\prime}$. Then there exist states $u^{\prime \prime \prime}$ and $u^{\prime}$ such that $u^{\prime \prime} \longrightarrow u^{\prime \prime \prime} \stackrel{(a)}{\longrightarrow} u^{\prime}, t^{\prime \prime} \mathcal{R}_{2} u^{\prime \prime \prime}$ and $t^{\prime} \mathcal{R}_{2} u^{\prime}$. So, $u \longrightarrow u^{\prime \prime \prime} \stackrel{(a)}{\longrightarrow} u^{\prime}$, from $s \mathcal{R}_{1} t^{\prime \prime}$ and $t^{\prime \prime} \mathcal{R}_{2} u^{\prime \prime \prime}$ it follows that $s \mathcal{R} u^{\prime \prime \prime}$, and from $s^{\prime} \mathcal{R}_{1} t^{\prime}$ and $t^{\prime} \mathcal{R}_{2} u^{\prime}$ it follows that $s^{\prime} \mathcal{R} u^{\prime}$.

(ii) To prove that $\mathcal{R}$ satisfies $\left(\overline{\mathrm{D}_{3}}\right)$, suppose that $s \mathcal{R} u$ and that there is an infinite sequence of states $\left(s_{k}\right)_{k \in \omega}$ such that $s=s_{0}, s_{k} \stackrel{\tau}{\longrightarrow} s_{k+1}$ for all $k \in \omega$. As before, there exists a state $t$ such that $s \mathcal{R}_{1} t$ and $t \mathcal{R}_{2} u$. From $s \mathcal{R}_{1} t$ it follows that there exist an infinite sequence of states $\left(t_{\ell}\right)_{\ell \in \omega}$ and a mapping $\sigma: \omega \rightarrow \omega$ such that $t=t_{0}, t_{\ell} \stackrel{\tau}{\longrightarrow} t_{\ell+1}$ and $s_{\sigma(\ell)} \mathcal{R} t_{\ell}$ for all $\ell \in \omega$. Hence, since $t \mathcal{R}_{2} u$, it follows that there exist an infinite sequence of states $\left(u_{m}\right)_{m \in \omega}$ and a mapping $\rho: \omega \rightarrow \omega$ such that $u=u_{0}, u_{m} \stackrel{\tau}{\longrightarrow} u_{m+1}$ and $t_{\rho(m)} \mathcal{R}_{2} u_{m}$ for all $m \in \omega$. Clearly, $s_{\sigma(\rho(m))} \mathcal{R} u_{m}$ for all $m \in \omega$.

Theorem 4.1. $\overleftrightarrow{b}_{b}^{\Delta_{3}}$ is an equivalence.

Proof The diagonal on $S$ (i.e., the binary relation $\{(s, s) \mid s \in S\}$ ) is a symmetric relation that satisfies

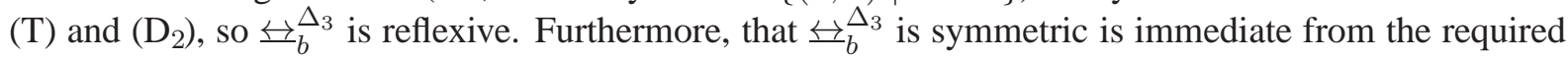
symmetry of the witnessing relation.

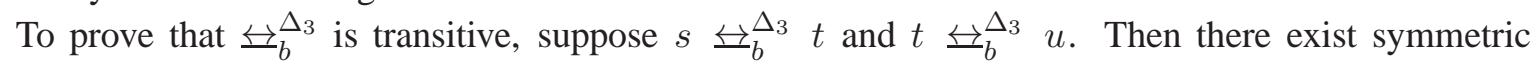
binary relations $\mathcal{R}_{1}$ and $\mathcal{R}_{2}$ satisfying (T) and $\left(\overline{\mathrm{D}_{3}}\right)$ such that $s \mathcal{R}_{1} t$ and $t \mathcal{R}_{2} u$. The relation $\mathcal{R}=$ $\left(\mathcal{R}_{1} ; \mathcal{R}_{2}\right) \cup\left(\mathcal{R}_{2} ; \mathcal{R}_{1}\right)$ is clearly symmetric and, by Lemmas 4.1 and 4.3 , satisfies $(\mathrm{T})$ and $\left(\mathrm{D}_{3}\right)$. Hence,

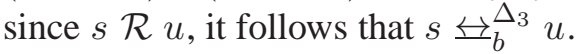

\section{2. $\unlhd_{b}^{\Delta_{3}}$ is included in $\bigsqcup_{b}^{\Delta}$}

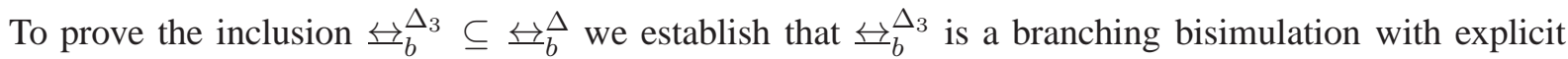
divergence.

Lemma 4.4. The relation $\overleftrightarrow{ }_{b}^{\Delta_{3}}$ satisfies (T) and $\left(\overline{D_{3}}\right)$.

Proof Directly from the definition it follows that $\overleftrightarrow{b}_{b}^{\Delta_{3}}$ is the union of all symmetric relations satisfying

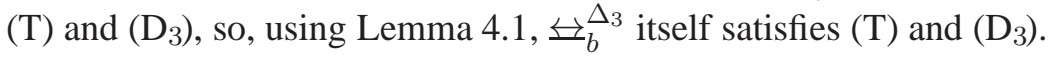

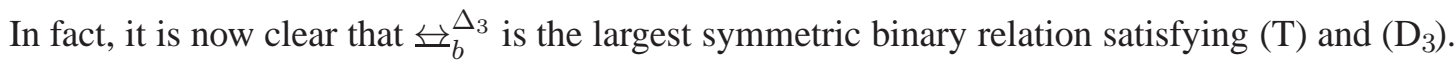

Lemma 4.5. The relation $\overleftrightarrow{b}_{b}^{\Delta_{3}}$ satisfies (D).

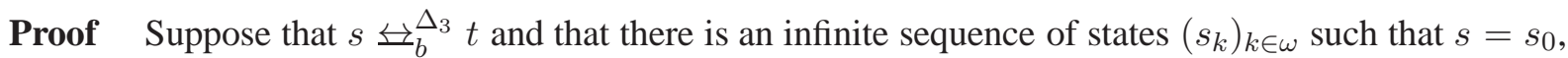
$s_{k} \stackrel{\tau}{\longrightarrow} s_{k+1}$ and $s_{k} \overleftrightarrow{ }_{b}^{\Delta_{3}} t$ for all $k \in \omega$. According to Lemma 4.4, the relation $\overleftrightarrow{ }_{b}^{\Delta_{3}}$ satisfies $\left(\overline{\mathrm{D}_{3}}\right)$, so there exist an infinite sequence of states $\left(t_{\ell}\right)_{\ell \in \omega}$ and a mapping $\sigma: \omega \rightarrow \omega$ such that $t=t_{0}, t_{\ell} \stackrel{\tau}{\longrightarrow} t_{\ell+1}$

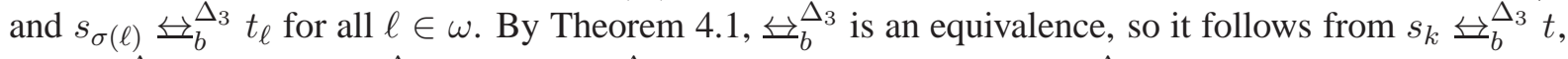

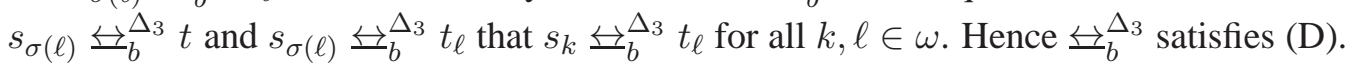




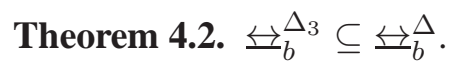

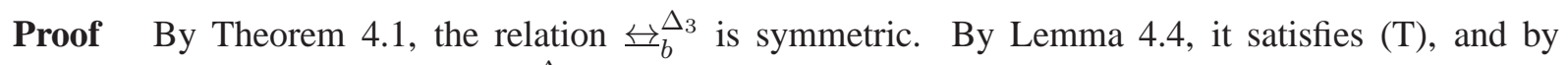

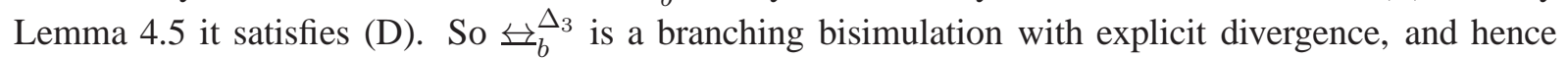

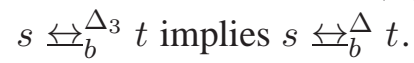

\subsection{Stuttering closure}

Definition 4.1. A binary relation $\mathcal{R}$ has the stuttering property if, whenever $t_{0} \stackrel{\tau}{\longrightarrow} \cdots \stackrel{\tau}{\longrightarrow} t_{n}, s \mathcal{R} t_{0}$ and $s \mathcal{R} t_{n}$, then $s \mathcal{R} t_{i}$ for all $i=0, \ldots, n$.

The following operation converts any binary relation $\mathcal{R}$ on $S$ into a larger relation $\hat{\mathcal{R}}$ that has the stuttering property.

Definition 4.2. Let $\mathcal{R}$ be a binary relation on $S$. The stuttering closure $\hat{\mathcal{R}}$ of $\mathcal{R}$ is defined by

$$
\hat{\mathcal{R}}=\left\{(s, t) \mid \exists s^{b}, s^{\sharp}, t^{b}, t^{\sharp} \in S \cdot s^{b} \longrightarrow s \longrightarrow s^{\sharp} \& t^{b} \longrightarrow t \longrightarrow t^{\sharp} \& s^{b} \mathcal{R} t^{\sharp} \& s^{\sharp} \mathcal{R} t^{b}\right\} .
$$

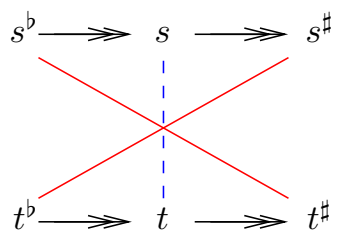

Figure 10. Stuttering closure.

Figure 10 illustrates the notion of stuttering closure. Clearly $\mathcal{R} \subseteq \hat{\mathcal{R}}$. We establish a few basic properties of the stuttering closure.

Lemma 4.6. The stuttering closure of a binary relation has the stuttering property.

Proof Let $\mathcal{R}$ be a binary relation and let $\hat{\mathcal{R}}$ be its stuttering closure. To show that $\hat{\mathcal{R}}$ has the stuttering property, suppose that $t_{0} \stackrel{\tau}{\longrightarrow} \cdots \stackrel{\tau}{\longrightarrow} t_{n}, s \hat{\mathcal{R}} t_{0}$ and $s \hat{\mathcal{R}} t_{n}$. Then, on the one hand, there exist states $s^{\sharp}$ and $t_{0}^{b}$ such that $s \longrightarrow s^{\sharp}, t_{0}^{b} \longrightarrow t_{0}$ and $s^{\sharp} \mathcal{R} t_{0}^{b}$, and on the other hand there exist states $s^{b}$ and $t_{n}^{\sharp}$ such that $s^{b} \longrightarrow s, t_{n} \longrightarrow t_{n}^{\sharp}$ and $s^{b} \mathcal{R} t_{n}^{\sharp}$. Now, since $s^{b} \longrightarrow s \longrightarrow s^{\sharp}$ and $t_{0}^{b} \longrightarrow t_{i} \longrightarrow t_{n}^{\sharp}$ for all $i=0, \ldots, n$, it follows that $s \hat{\mathcal{R}} t_{i}$.

Remark 4.1. The stuttering closure $\hat{\mathcal{R}}$ of a binary relation $\mathcal{R}$ is (contrary to what our terminology may suggest) not necessarily the smallest relation containing $\mathcal{R}$ with the stuttering property. For a counterexample, consider a transition system with states $s^{b}, s^{\sharp}, t^{b}$ and $t^{\sharp}$ and transitions $s^{b} \stackrel{\tau}{\longrightarrow} s^{\sharp}$ and $t^{b} \stackrel{\tau}{\longrightarrow} t^{\sharp}$; the binary relation

$$
\mathcal{R}=\left\{\left(s^{b}, t^{\sharp}\right),\left(t^{\sharp}, s^{b}\right),\left(s^{\sharp}, t^{b}\right),\left(t^{b}, s^{\sharp}\right),\left(s^{\sharp}, t^{\sharp}\right),\left(t^{\sharp}, s^{\sharp}\right)\right\}
$$

has the stuttering property, but $\hat{\mathcal{R}}$ has additionally the pairs $\left(s^{b}, t^{b}\right)$ and $\left(t^{b}, s^{b}\right)$. 
Lemma 4.7. The stuttering closure $\hat{\mathcal{R}}$ of a symmetric binary relation $\mathcal{R}$ is symmetric.

Proof Suppose $s \hat{\mathcal{R}} t$; then there exist states $s^{b}, s^{\sharp}, t^{b}$ and $t^{\sharp}$ such that $s^{b} \longrightarrow s \longrightarrow s^{\sharp}, t^{b} \longrightarrow t \longrightarrow t^{\sharp}$, $s^{b} \mathcal{R} t^{\sharp}$ and $s^{\sharp} \mathcal{R} t^{b}$. Since $\mathcal{R}$ is symmetric, it follows that $t^{b} \mathcal{R} s^{\sharp}$ and $t^{\sharp} \mathcal{R} s^{b}$. Hence $t \hat{\mathcal{R}} s$.

Lemma 4.8. Let $\hat{\mathcal{R}}$ be the stuttering closure of $\mathcal{R}$. If $\mathcal{R}$ satisfies $(T)$ and $s \hat{\mathcal{R}} t$, then there exists $t^{\prime}$ such that $t \longrightarrow t^{\prime}$ and $s \mathcal{R} t^{\prime}$.

Proof Suppose $s \hat{\mathcal{R}} t$; then there exist states $s^{b}, s^{\sharp}, t^{b}$ and $t^{\sharp}$ such that $s^{b} \longrightarrow s \longrightarrow s^{\sharp}, t^{b} \longrightarrow t \longrightarrow t^{\sharp}$, $s^{b} \mathcal{R} t^{\sharp}$ and $s^{\sharp} \mathcal{R} t^{b}$. From $s^{b} \mathcal{R} t^{\sharp}$ and $s^{b} \longrightarrow s$ it follows by Lemma 4.2 that there exists $t^{\prime}$ such that $(t \longrightarrow) t^{\sharp} \longrightarrow t^{\prime}$ and $s \mathcal{R} t^{\prime}$.

Lemma 4.9. If $\mathcal{R}$ satisfies $[\mathrm{T}$, then so does its stuttering closure $\hat{\mathcal{R}}$.

Proof Suppose that $s \hat{\mathcal{R}} t$ and that $s \stackrel{a}{\longrightarrow} s^{\prime}$ for some $s^{\prime}$. Then by Lemma 4.8 there exists $t^{\dagger}$ such that $t \longrightarrow t^{\dagger}$ and $s \mathcal{R} t^{\dagger}$. Hence, since $s \stackrel{a}{\longrightarrow} s^{\prime}$, it follows by (T) that there exist states $t^{\prime \prime}$ and $t^{\prime}$ such that

$$
(t \longrightarrow) t^{\dagger} \longrightarrow t^{\prime \prime} \stackrel{(a)}{\longrightarrow} t^{\prime}, s \mathcal{R} t^{\prime \prime} \text { and } s^{\prime} \mathcal{R} t^{\prime}
$$

Now, $s \mathcal{R} t^{\prime \prime}$ and $s^{\prime} \mathcal{R} t^{\prime}$ respectively imply $s \hat{\mathcal{R}} t^{\prime \prime}$ and $s^{\prime} \hat{\mathcal{R}} t^{\prime}$.

\subsection{Closing the cycle of inclusions}

Using the notion of stuttering closure we can now prove $\unlhd_{b}^{\Delta_{4}} \subseteq \overleftrightarrow{B}_{b}^{\Delta_{3}}$, thereby closing the cycle of

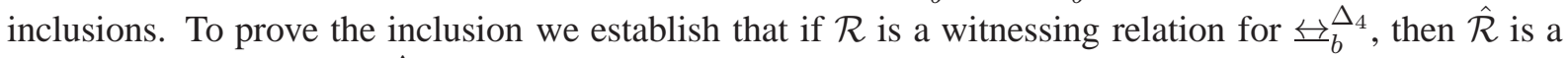

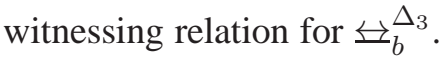

Lemma 4.10. If $\mathcal{R}$ satisfies $(\mathrm{T})$ and $\left(\overline{\mathrm{D}_{4}}\right)$, then $\hat{\mathcal{R}}$ satisfies $\left(\mathrm{D}_{3}\right)$.

Proof Suppose that $\mathcal{R}$ satisfies (T) and $\left(\mathrm{D}_{4}\right)$. By Proposition 3.1 it suffices to establish that $\hat{\mathcal{R}}$ satisfies $\left(\overline{\mathrm{D}_{2}}\right)$. Suppose that $s \hat{\mathcal{R}} t$ and there exists an infinite sequence of states $\left(s_{k}\right)_{k \in \omega}$ such that $s=s_{0}$ and $s_{k} \stackrel{\tau}{\longrightarrow} s_{k+1}$ for all $k \in \omega$. We have to show that there exists a state $t^{\prime}$ such that $t \stackrel{\tau}{\longrightarrow} t^{\prime}$ and $s_{k} \hat{\mathcal{R}} t^{\prime}$ for some $k \in \omega$.

As $s \hat{\mathcal{R}} t$, by Lemma 4.8 there exist $t_{0}, \ldots, t_{n}$ such that $t=t_{0} \stackrel{\tau}{\longrightarrow} \cdots \stackrel{\tau}{\longrightarrow} t_{n}$ and $s \mathcal{R} t_{n}$. By Lemma 4.6, $s \hat{\mathcal{R}} t_{i}$ for all $i=0, \ldots, n$, so if $n>0$, then we can take $t^{\prime}=t_{1}$. We proceed with the assumption that $n=0$; so $s \mathcal{R} t$.

First suppose that $s_{k} \mathcal{R} t$ for all $k \in \omega$. Then by condition $\left(\overline{\mathrm{D}_{4}}\right)$ there exist $t_{0}, \ldots, t_{m}$ such that $t=t_{0} \stackrel{\tau}{\longrightarrow} \cdots \stackrel{\tau}{\longrightarrow} t_{m}$ with $m>0$ and $s_{k} \mathcal{R} t_{m}$ for some $k \in \omega$. As $s_{k} \hat{\mathcal{R}} t_{0}$ and $s_{k} \hat{\mathcal{R}} t_{m}$, it follows by Lemma 4.6 that $s_{k} \hat{\mathcal{R}} t_{i}$ for all $i=0, \ldots, n$. Hence, in particular, $s_{k} \hat{\mathcal{R}} t_{1}$, so we can take $t^{\prime}=t_{1}$.

In the remaining case there is a $k_{0} \in \omega$ such that $s_{k} \mathcal{R} t$ for all $k \leq k_{0}$ while $s_{k_{0}+1}$ and $t$ are not related by $\mathcal{R}$. Since $s_{k_{0}} \mathcal{R} t$ and $s_{k_{0}} \stackrel{\tau}{\longrightarrow} s_{k_{0}+1}$, by condition (T) of Definition 3.1 there exist states $t_{0}, \ldots, t_{m}, t_{m+1}$ such that $t=t_{0} \stackrel{\tau}{\longrightarrow} \cdots \stackrel{\tau}{\longrightarrow} t_{m} \stackrel{(\tau)}{\longrightarrow} t_{m+1}, s_{k_{0}} \mathcal{R} t_{m}$ and $s_{k_{0}+1} \mathcal{R} t_{m+1}$. Since $s_{k_{0}+1}$ and $t$ are not related by $\mathcal{R}$, it follows that $t_{0} \neq t_{m+1}$, so either $m>0$ or $t_{m} \stackrel{\tau}{\longrightarrow} t_{m+1}$. In case $m>0$, since $s_{k_{0}} \hat{\mathcal{R}} t_{0}$ and $s_{k_{0}} \hat{\mathcal{R}} t_{m}$, by Lemma 4.6 it follows that $s_{k_{0}} \hat{\mathcal{R}} t_{1}$, so we can take $t^{\prime}=t_{1}$. In case $m=0$ and $t=t_{m} \stackrel{\tau}{\longrightarrow} t_{m+1}$, we can take $t^{\prime}=t_{m+1}$. 


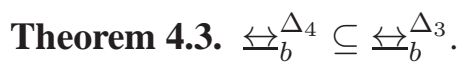

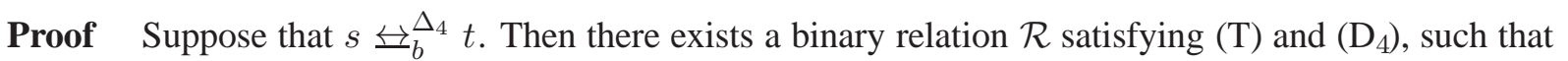
$s \mathcal{R} t$. By Lemma 4.7 the stuttering closure $\hat{\mathcal{R}}$ of $\mathcal{R}$ is symmetric, by Lemma 4.9 it satisfies $(\mathrm{T})$, and by Lemma 4.10 it satisfies $\left(\mathrm{D}_{3}\right)$. Moreover, $s \hat{\mathcal{R}} t$. Hence, $s \overleftrightarrow{\longrightarrow}_{b}^{\Delta_{3}} t$.

The inclusions already established in Section 3 together with the inclusions established in Theorems 4.2 and 4.3 yield the following corollary (see also Figure 9 ).

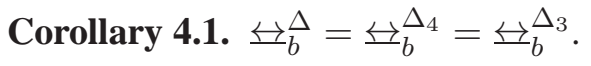

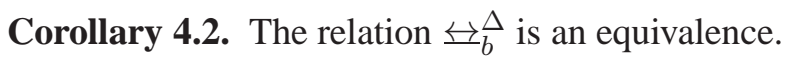

Recall that the proof strategy employed in Lemma 4.1) (ii) to show that any union of binary relations satisfying $\left(\overline{D_{3}}\right)$ also satisfies $\left(\overline{D_{3}}\right)$, fails with $(\mathrm{D})$ or $\left(\overline{D_{4}}\right)$ instead of $\left(\overline{D_{3}}\right)$. In fact, it is easy to show that

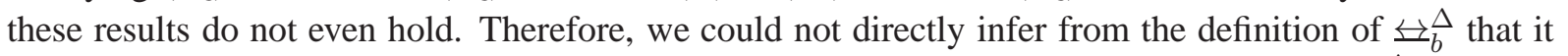

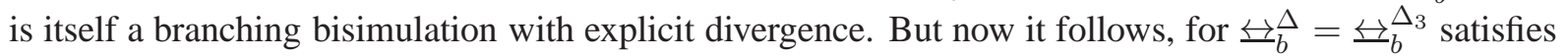
$(\mathrm{T})$ and $\left(\mathrm{D}_{3}\right)$ by Lemma 4.4 and hence also the weaker condition $\left(\mathrm{D}_{4}\right)$. It satisfies $(\mathrm{D})$ by Lemma 4.5

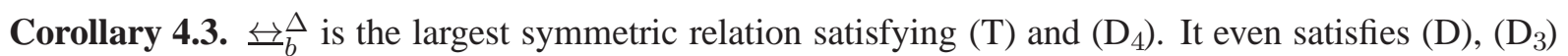
and $\left(\mathrm{D}_{2}\right)$. It therefore is the largest branching bisimulation with explicit divergence.

The following corollary is another consequence, which we need in the next section.

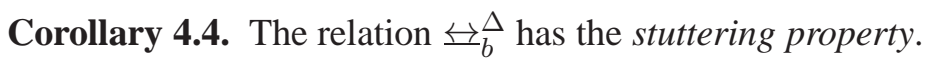

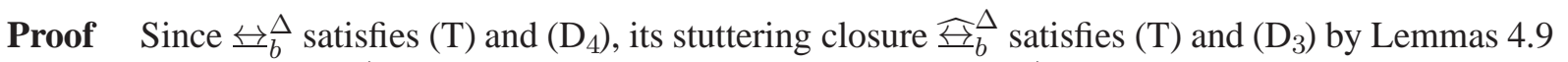

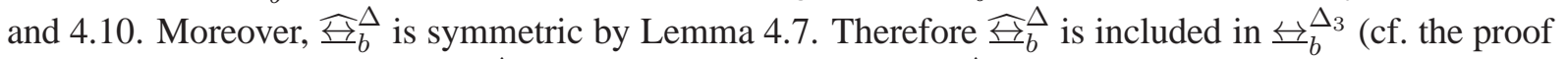

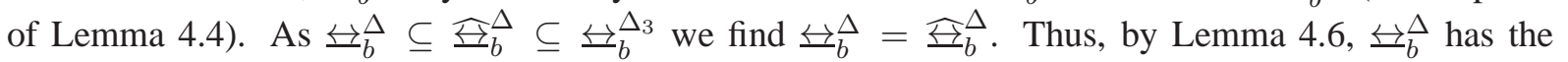
stuttering property.

\section{Coloured-trace characterisation of $\mathbf{B B}^{\Delta}$}

We now recall from [6] the original characterisation in terms of coloured traces of branching bisimilarity with explicit divergence, and establish that it coincides with the relational characterisations of Section 3 .

Definition 5.1. Let $\mathcal{C}$ be a colouring. A state $s$ is $\mathcal{C}$-divergent if there exists an infinite sequence of states $\left(s_{k}\right)_{k \in \omega}$ such that $s=s_{0}, s_{k} \stackrel{\tau}{\longrightarrow} s_{k+1}$ and $\mathcal{C}\left(s_{k}\right)=\mathcal{C}(s)$ for all $k \in \omega$. A consistent colouring is said to preserve divergence if no $\mathcal{C}$-divergent state has the same colour as a nondivergent state.

We write $s \equiv_{c}^{\Delta} t$ if there exists a consistent, divergence preserving colouring $\mathcal{C}$ with $\mathcal{C}(s)=\mathcal{C}(t)$.

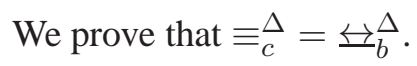

Lemma 5.1. Let $\mathcal{C}$ be a colouring such that two states with the same colour have the same $\mathcal{C}$-coloured traces of length three (i.e. colour - action - colour). Then $\mathcal{C}$ is consistent. 
Proof Suppose $\mathcal{C}\left(s_{0}\right)=\mathcal{C}\left(t_{0}\right)$ and $C_{0}, a_{1}, C_{1}, \ldots, a_{n}, C_{n}$ is a coloured trace of $s_{0}$. Then, for $i=$ $1, \ldots, n$, there are states $s_{i}$ and paths $\pi_{i}$ from $s_{i-1}$ to $s_{i}$, such that $\mathcal{C}\left(\pi_{i}\right)=C_{i-1}, a_{i}, C_{i}$. With induction on $i$, for $i=1, \ldots, n$ we find states $t_{i}$ with $C\left(s_{i}\right)=C\left(t_{i}\right)$ and paths $\rho_{i}$ from $t_{i-1}$ to $t_{i}$ such that $\mathcal{C}\left(\rho_{i}\right)=$ $C_{i-1}, a_{i}, C_{i}$. Namely, the assumption about $\mathcal{C}$ allows us to find $\rho_{i}$ given $t_{i-1}$, and then $t_{i}$ is defined as the last state of $\rho_{i}$. Concatenating all the paths $\rho_{i}$ yields a path $\rho$ from $t_{0}$ with $\mathcal{C}(\rho)=C_{0}, a_{1}, C_{1}, \ldots, a_{n}, C_{n}$.

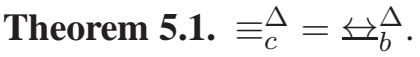

Proof " $\subseteq$ ": Let $\mathcal{C}$ be a consistent colouring that preserves divergence. It suffices to show that $\mathcal{C}$ is a branching bisimulation with explicit divergence.

Suppose $s \mathcal{C} t$, i.e. $\mathcal{C}(s)=\mathcal{C}(t)$, and $s \stackrel{a}{\longrightarrow} s^{\prime}$ for some state $s^{\prime}$. In case $a=\tau$ and $\mathcal{C}\left(s^{\prime}\right)=\mathcal{C}(s)$ we have $s^{\prime} \mathcal{C} t$ and condition (T) is satisfied. So suppose $a \neq \tau$ or $\mathcal{C}\left(s^{\prime}\right) \neq \mathcal{C}(s)$. Then $s$, and therefore also $t$, has a coloured trace $\mathcal{C}(s), a, \mathcal{C}\left(s^{\prime}\right)$. This implies that there are states $t_{0}, \ldots, t_{n}$ for some $n \geq 0$ and $t^{\prime}$ with $t=t_{0} \stackrel{\tau}{\longrightarrow} t_{1} \stackrel{\tau}{\longrightarrow} \cdots \stackrel{\tau}{\longrightarrow} t_{n} \stackrel{(a)}{\longrightarrow} t^{\prime}$ such that $\mathcal{C}\left(t_{i}\right)=\mathcal{C}(s)$ for $i=0, \ldots, n$ and $\mathcal{C}\left(t^{\prime}\right)=\mathcal{C}\left(s^{\prime}\right)$. Hence (T) is satisfied.

Now suppose $s \mathcal{C} t$ and there is an infinite sequence of states $\left(s_{k}\right)_{k \in \omega}$ such that $s=s_{0}, s_{k} \stackrel{\tau}{\longrightarrow} s_{k+1}$ and $s_{k} \mathcal{C} t$ for all $k \in \omega$. Then $\mathcal{C}\left(s_{k}\right)=\mathcal{C}(s)$ for all $k \in \omega$. Hence $s$, and therefore also $t$, is $\mathcal{C}$-divergent. Thus there exists an infinite sequence of states $\left(t_{\ell}\right)_{\ell \in \omega}$ such that $t=t_{0}, t_{\ell} \stackrel{\tau}{\longrightarrow} t_{\ell+1}$ and $\mathcal{C}\left(t_{\ell}\right)=\mathcal{C}(t)$ for all $\ell \in \omega$. It follows that $s_{k} \mathcal{C} t_{\ell}$ for all $k, \ell \in \omega$. Hence also (D) is satisfied.

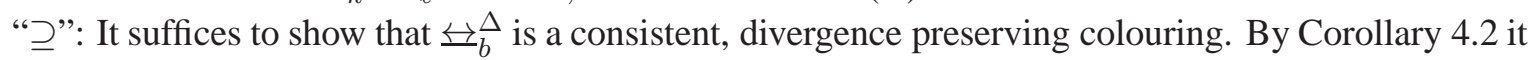
is an equivalence. We also use that it satisfies (T) and (D) (Corollary 4.3) and has the stuttering property (Corollary 4.4). We invoke Lemma 5.1 for proving consistency.

Suppose that $s$ and $t$ have the same colour, i.e., $s \unlhd_{b}^{\Delta} t$, and let $C, a, D$ be a $\unlhd_{b}^{\Delta}$-coloured trace of $s$. Then $a \neq \tau$ or $C \neq D$, and there are states $s^{\prime \prime}$ and $s^{\prime}$ with $s \longrightarrow s^{\prime \prime} \stackrel{a}{\longrightarrow} s^{\prime}$, such that $s^{\prime \prime}, s \in C$ and

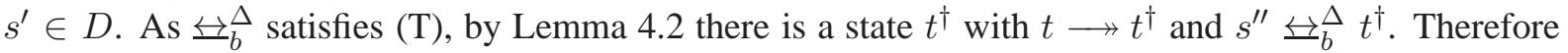

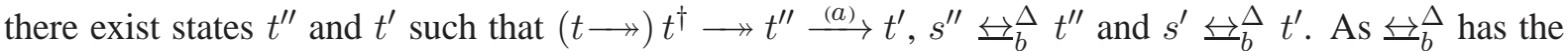

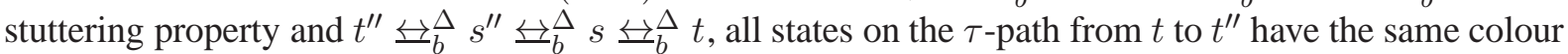

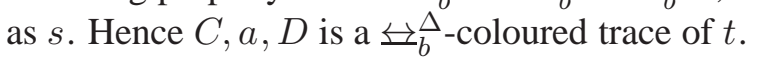

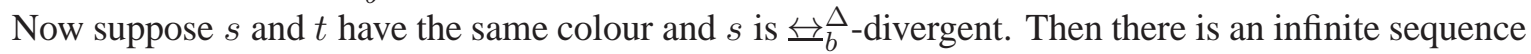

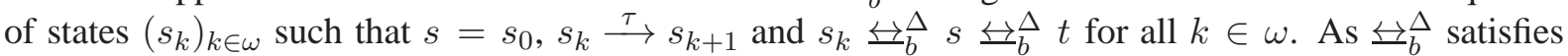
(D), this implies that there exists an infinite sequence of states $\left(t_{\ell}\right)_{\ell \in \omega}$ such that $t=t_{0}, t_{\ell} \stackrel{\tau}{\longrightarrow} t_{\ell+1}$ and

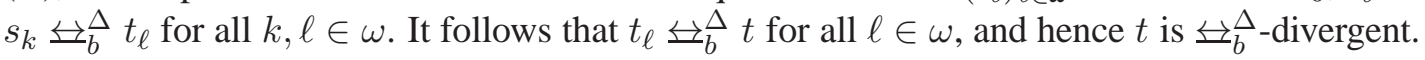

\section{Modal characterisations of $\mathbf{B B}^{\Delta}$}

We shall now establish agreement between the relational and modal characterisations of $\mathrm{BB}^{\Delta}$ proposed in [4]. The class of formulas $\Phi_{j b}^{\Delta}$ of the modal logic for $\mathrm{BB}^{\Delta}$ proposed in [4] is generated by the grammar obtained by adding the following clause to the grammar in (1) of Section 2 .

$$
\varphi::=\Delta \varphi \quad\left(\varphi \in \Phi_{j b}^{\Delta}\right) .
$$

We extend the inductive definition of validity in Section 2 with: 
(iv) $s \models \Delta \varphi$ iff there exists an infinite sequence $\left(s_{k}\right)_{k \in \omega}$ of states such that $s \longrightarrow s_{0}, s_{k} \stackrel{\tau}{\longrightarrow} s_{k+1}$ and $s_{k}=\varphi$ for all $k \in \omega$.

Again, validity induces an equivalence on states: we define $\approx^{\Delta} \subseteq S \times S$ by

$$
s \approx^{\Delta} t \quad \text { iff } \quad \forall \varphi \in \Phi_{j b}^{\Delta} . s \models \varphi \Leftrightarrow t \models \varphi .
$$

We shall now establish that $\approx^{\Delta}$ coincides with $\bigsqcup_{b}^{\Delta}$.

Theorem 6.1. For all states $s$ and $t: s \unlhd_{b}^{\Delta} t$ iff $s \approx^{\Delta} t$.

Proof To establish the implication from left to right, we prove by structural induction on $\varphi$ that if

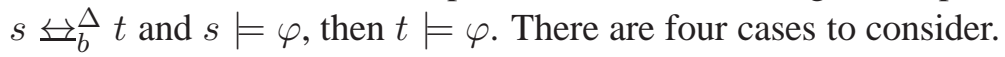

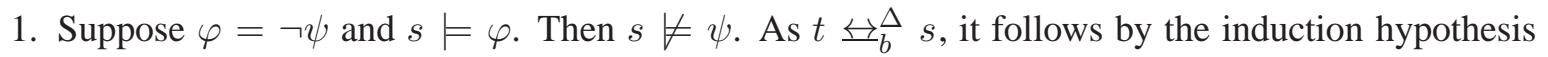
that $t \not \models \psi$, and hence $t \models \varphi$.

2. Suppose $s \models \bigwedge \Psi$. Then, for all $\psi \in \Psi, s \models \psi$, and by induction $t \models \psi$. This yields $t \models \phi$.

3. Suppose $\varphi=\psi a \chi$ and $s \models \varphi$. Then there exist states $s^{\prime}$ and $s^{\prime \prime}$ such that $s \longrightarrow s^{\prime \prime} \stackrel{(a)}{\longrightarrow} s^{\prime}, s^{\prime \prime} \models \psi$

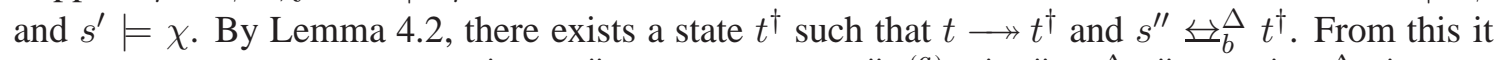

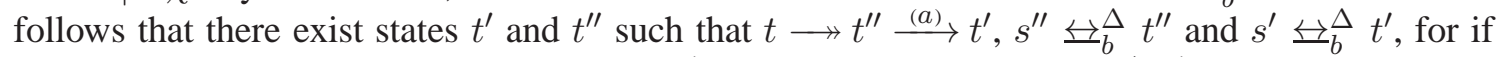

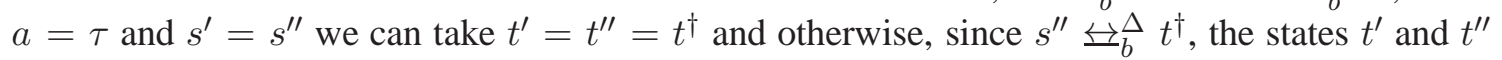
exist by (T). It follows by the induction hypothesis that $t^{\prime \prime} \models \psi$ and $t^{\prime} \models \chi$, and hence $t \models \varphi$.

4. Suppose $\varphi=\Delta \psi$ and $s \models \varphi$. Then there exists an infinite sequence $\left(s_{k}\right)_{k \in \omega}$ such that $s \longrightarrow s_{0}$, $s_{k} \stackrel{\tau}{\longrightarrow} s_{k+1}$ and $s_{k} \models \psi$ for all $k \in \omega$. By Lemma 4.2 there exists a state $t_{0}$ such that $t \longrightarrow t_{0}$ and

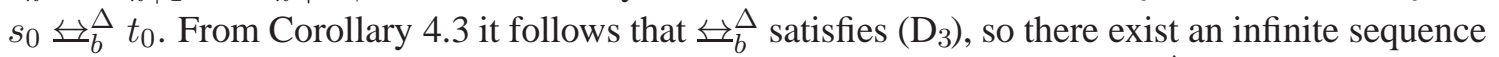
of states $\left(t_{\ell}\right)_{\ell \in \omega}$ and a mapping $\sigma: \omega \rightarrow \omega$ such that $t_{\ell} \stackrel{\tau}{\longrightarrow} t_{\ell+1}$ and $s_{\sigma(\ell)} \overleftrightarrow{\longrightarrow}_{b}^{\Delta} t_{\ell}$ for all $\ell \in \omega$. By the induction hypothesis $t_{\ell} \models \psi$ for all $\ell \in \omega$, and hence $t \models \varphi$.

For the implication from right to left, it suffices by Corollary 4.1 to prove that $\approx^{\Delta}$ is symmetric and satisfies the conditions $(\mathrm{T})$ and $\left(\overline{\mathrm{D}_{4}}\right)$.

That $\approx^{\Delta}$ is symmetric is clear from its definition.

To establish condition (T) of Definition 3.1, suppose that $s \approx^{\Delta} t$ and $s \stackrel{a}{\longrightarrow} s^{\prime}$, and define sets $T^{\prime \prime}$ and $T^{\prime}$ as follows:

$$
\begin{aligned}
& T^{\prime \prime}=\left\{t^{\prime \prime} \in S \mid t \longrightarrow t^{\prime \prime} \& s \not^{\Delta} t^{\prime \prime}\right\} ; \text { and } \\
& T^{\prime}=\left\{t^{\prime} \in S \mid \exists t^{\prime \prime} \in S . t \longrightarrow t^{\prime \prime} \stackrel{(a)}{\longrightarrow} t^{\prime} \& s^{\prime} \not^{\Delta} t^{\prime}\right\} .
\end{aligned}
$$

For each $t^{\prime \prime} \in T^{\prime \prime}$ let $\varphi_{t^{\prime \prime}}$ be a formula such that $s \models \varphi_{t^{\prime \prime}}$ and $t^{\prime \prime} \not \models \varphi_{t^{\prime \prime}}$, and let $\varphi=\bigwedge\left\{\varphi_{t^{\prime \prime}} \mid t^{\prime \prime} \in T^{\prime \prime}\right\}$. Similarly, for each $t^{\prime} \in T^{\prime}$ let $\psi_{t^{\prime}}$ be a formula with $s^{\prime} \models \psi_{t^{\prime}}$ and $t^{\prime} \not \models \psi_{t^{\prime}}$, and let $\psi=\bigwedge\left\{\psi_{t^{\prime}} \mid t^{\prime} \in T^{\prime}\right\}$. Note that $s \models \varphi a \psi$, and hence, since $s \approx^{\Delta} t$, also $t \models \varphi a \psi$. So, there exist states $t^{\prime}$ and $t^{\prime \prime}$ such that $t \longrightarrow t^{\prime \prime} \stackrel{(a)}{\longrightarrow} t^{\prime}, t^{\prime \prime} \models \varphi$ and $t^{\prime} \models \psi$. From $t^{\prime \prime} \models \varphi$ it follows that $t^{\prime \prime} \notin T^{\prime \prime}$, so $s \approx^{\Delta} t^{\prime \prime}$; from $t^{\prime} \models \psi$ it follows that $t^{\prime} \notin T^{\prime}$, so $s^{\prime} \approx^{\Delta} t^{\prime}$. Thereby, condition (T) is established.

To establish condition $\left(\mathrm{D}_{4}\right)$, suppose that $s \approx^{\Delta} t$ and that there exists an infinite sequence $\left(s_{k}\right)_{k \in \omega}$ such that $s=s_{0}, s_{k} \stackrel{\tau}{\longrightarrow} s_{k+1}$ and $s_{k} \approx^{\Delta} t$ for all $k \in \omega$. Define the set $T^{\infty}$ by

$$
T^{\infty}=\left\{t^{\prime} \in S \mid t \longrightarrow t^{\prime} \& s \not^{\Delta} t^{\prime}\right\} .
$$


For each $t^{\prime} \in T^{\infty}$ let $\varphi_{t^{\prime}}$ be a formula such that $s \models \varphi_{t^{\prime}}$ and $t^{\prime} \not \models \varphi_{t^{\prime}}$, and let $\varphi=\bigwedge\left\{\varphi_{t^{\prime}} \mid t^{\prime} \in T^{\infty}\right\}$. Since $s_{0}=s \models \varphi$ and $s_{k} \approx^{\Delta} t \approx^{\Delta} s$, it follows that $s_{k} \models \varphi$ for all $k \in \omega$, and therefore $s \models \Delta \varphi$. Hence, $t \models \Delta \varphi$, so there exists an infinite sequence $\left(t_{\ell}\right)_{\ell \in \omega}$ such that $t \longrightarrow t_{0}, t_{\ell} \stackrel{\tau}{\longrightarrow} t_{\ell+1}$ and $t_{\ell} \models \varphi$ for all $\ell \in \omega$. It follows that $t_{\ell} \notin T^{\infty}$, so $s \approx^{\Delta} t_{\ell}$, for all $\ell \in \omega$, and hence $s_{k} \approx^{\Delta} s \approx^{\Delta} t_{\ell}$ for all $k, \ell \in \omega$. It follows, in particular, that $t \longrightarrow^{+} t_{1}$ and $s_{k} \approx^{\Delta} t_{1}$ for some $k \in \omega$. Thereby, also condition ( $\left(\mathrm{D}_{4}\right)$ is established.

We already mentioned in Section 2 the result of Laroussinie, Pinchinat \& Schnoebelen [8] that the modal logic with negation, binary conjunction and $\langle\hat{a}\rangle$ and the logic with negation, binary conjunction and $a$ are equally expressive. Below, we adapt their method to show that replacing $a$ by $\langle\hat{a}\rangle$ or $\langle a\rangle$ in the modal logic for $\mathrm{BB}^{\Delta}$ proposed in [4] also yields an equally expressive logic.

Henceforth we denote by $\Phi_{u}^{\Delta}$ the set of formulas generated by the grammar that is obtained when replacing $\varphi a \varphi$ by $\varphi\langle a\rangle \varphi$ in the grammar for $\Phi_{j b}^{\Delta}$ (see (1) in Section 2 and (2) at the beginning of this section). The central idea, from [8], is that any formula in $\Phi_{j b}^{\Delta}$ can be written as a Boolean combination of formulas that propagate either upwards or downwards along a path of $\tau$-transitions. A formula $\varphi$ that propagates upwards, i.e., with the property that if $s \longrightarrow t$ and $s \models \varphi$, then also $t \models \varphi$, we shall call an upward formula. A formula $\varphi$ that propagates downwards, i.e., with the property that if $s \longrightarrow t$ and $t \models \varphi$, then also $s \models \varphi$, we shall call a downward formula.

Lemma 6.1. Every $\varphi \in \Phi_{j b}^{\Delta}$ is equivalent with a formula of the form $\bigvee \Phi$, where each formula in $\Phi$ is a conjunction of an upward and a downward formula.

Proof Note that $\psi a \chi$ and $\Delta \psi$ are downward formulas and that the negation of a downward formula is an upward formula. Furthermore, a conjunction of upward formulas is again an upward formula and a conjunction of downward formulas is again a downward formula. It follows, by the standard laws of Boolean algebra, that the formula $\varphi$ is equivalent to a formula of the desired shape.

The proof that for every formula $\varphi \in \Phi_{u}^{\Delta}$ there exists an equivalent formula $\varphi^{\prime} \in \Phi_{j b}^{\Delta}$ proceeds by induction on the structure of $\varphi$, and the only nontrivial case is when $\varphi=\psi\langle a\rangle \chi$. According to the induction hypothesis, for $\psi$ and $\chi$ there exist equivalent formulas in $\Phi_{j b}^{\Delta}$, so, by Lemma 6.1 $\psi$ is equivalent to a disjunction of conjunctions of upward and downward formulas. The proof in [8] then relies on these disjunctions being finite. To generalise it to infinite disjunctions, we shall use the following lemma.

Lemma 6.2. Let $\Phi$ be a set of formulas and let $\varphi$ be a formula. Then

$$
(\bigvee \Phi)\langle a\rangle \varphi \Leftrightarrow \bigvee\left\{\left(\bigvee \Phi^{\prime}\right)\langle a\rangle \varphi \mid \Phi^{\prime} \text { a finite subset of } \Phi\right\}
$$

\section{Proof}

$(\Rightarrow)$ Suppose $s \models(\bigvee \Phi)\langle a\rangle \varphi$. Then there exist states $s_{0}, \ldots, s_{n}, s_{n+1}$ such that $s=s_{0} \stackrel{\tau}{\longrightarrow} \cdots \stackrel{\tau}{\longrightarrow}$ $s_{n} \stackrel{(a)}{\longrightarrow} s_{n+1}, s_{i} \models \bigvee \Phi$ for all $i=0, \ldots, n$ and $s_{n+1} \models \varphi$. Since $s_{i} \models \bigvee \Phi$, we can associate with every $s_{i}(i=0, \ldots, n)$ a formula $\varphi_{i} \in \Phi$ such that $s_{i} \models \varphi_{i}$. Let $\Phi^{\prime}=\left\{\varphi_{i} \mid i=0, \ldots, n\right\}$; then $\Phi^{\prime}$ is a finite subset of $\Phi$ such that $s_{i} \models \bigvee \Phi^{\prime}$ for every $i=0, \ldots, n$. It follows that $s \models\left(\bigvee \Phi^{\prime}\right)\langle a\rangle \varphi$, and hence $s \models \bigvee\left\{\left(\bigvee \Phi^{\prime}\right)\langle a\rangle \varphi \mid \Phi^{\prime}\right.$ a finite subset of $\left.\Phi\right\}$. 
$(\Leftarrow)$ If $s \models \bigvee\left\{\left(\bigvee \Phi^{\prime}\right)\langle a\rangle \varphi \mid \Phi^{\prime}\right.$ a finite subset of $\left.\Phi\right\}$, then $s \models\left(\bigvee \Phi^{\prime}\right)\langle a\rangle \varphi$ for some finite subset $\Phi^{\prime}$ of $\Phi$. So there exist states $s_{0}, \ldots, s_{n}, s_{n+1}$ such that $s=s_{0} \stackrel{\tau}{\longrightarrow} \cdots \stackrel{\tau}{\longrightarrow} s_{n} \stackrel{(a)}{\longrightarrow} s_{n+1}, s_{i} \models \bigvee \Phi^{\prime}$ for all $i=0, \ldots, n$ and $s_{n+1} \models \varphi$. Since $s_{i} \models \bigvee \Phi^{\prime}$ implies $s_{i} \models \bigvee \Phi$ for all $i=0, \ldots, n$, it follows that $s \models(\bigvee \Phi)\langle a\rangle \varphi$.

We now adapt the method in [8] and show that replacing $a$ by $\langle\hat{a}\rangle$ or $\langle a\rangle$ in the modal logic for $\mathrm{BB}^{\Delta}$ proposed in [4] yields an equally expressive logic.

Theorem 6.2. For every formula $\varphi \in \Phi_{u}^{\Delta}$ there exists an equivalent formula $\varphi^{\prime} \in \Phi_{j b}^{\Delta}$.

Proof The proof is by structural induction on $\varphi$; the only nontrivial case is when $\varphi=\psi\langle a\rangle \chi$. By the induction hypothesis there exist formulas $\psi^{\prime}, \chi^{\prime} \in \Phi_{j b}^{\Delta}$ such that $\psi \Leftrightarrow \psi^{\prime}$ and $\chi \Leftrightarrow \chi^{\prime}$. By Lemma6.1, $\psi^{\prime} \Leftrightarrow \bigvee \Psi$, where each formula in $\Psi$ is a conjunction of an upward and a downward formula. Hence, by the evident congruence property of $\Leftrightarrow$ and Lemma 6.2

$$
\varphi \Leftrightarrow \bigvee\left\{\left(\bigvee \Psi^{\prime}\right)\langle a\rangle \chi^{\prime} \mid \Psi^{\prime} \text { a finite subset of } \Psi\right\}
$$

Clearly, it now suffices to establish that $\left(\bigvee \Psi^{\prime}\right)\langle a\rangle \chi^{\prime}$ is equivalent to a formula in $\Phi_{j b}^{\Delta}$, for all finite subsets $\Psi^{\prime}$ of $\Psi$. Recall that $\Psi$ consists of conjunctions of an upward and a downward formula, so we can assume that $\Psi^{\prime}=\left\{\psi_{i}^{\mathrm{u}} \wedge \psi_{i}^{\mathrm{d}} \mid i=1, \ldots, n\right\}$; we proceed by induction on the cardinality of $\Psi^{\prime}$.

If $\left|\Psi^{\prime}\right|=0$, then

$$
\left(\bigvee \Psi^{\prime}\right)\langle a\rangle \chi^{\prime} \Leftrightarrow \perp
$$

and $\perp \in \Phi_{j b}^{\Delta}$.

Suppose $\left|\Psi^{\prime}\right|>0$. By the induction hypothesis there exists, for every $i=1, \ldots, n$, a formula $\varphi_{i}^{\prime} \in \Phi_{j b}^{\Delta}$ such that

$$
\left(\bigvee \Psi^{\prime}-\left\{\psi_{i}^{\mathrm{u}} \wedge \psi_{i}^{\mathrm{d}}\right\}\right)\langle a\rangle \chi^{\prime} \Leftrightarrow \varphi_{i}^{\prime}
$$

Then, it is easy to verify that

$$
\left(\bigvee \Psi^{\prime}\right)\langle a\rangle \chi^{\prime} \Leftrightarrow \bigvee_{i=1}^{n}\left(\psi_{i}^{\mathrm{u}} \wedge\left(\psi_{i}^{\mathrm{d}} a \chi^{\prime} \vee \psi_{i}^{\mathrm{d}} \tau \varphi_{i}^{\prime}\right)\right)
$$

and the right-hand side formula is in $\Phi_{j b}^{\Delta}$. Some intuition for this last step is offered in [8].

In the same vain, there is also an obvious strengthening of the divergence modality $\Delta$. Let $\widehat{\Delta}$ be the unary divergence modality with the following definition:

(iv $\left.^{\prime}\right) s \models \widehat{\Delta} \varphi$ iff there exists an infinite sequence $\left(s_{k}\right)_{k \in \omega}$ of states such that $s=s_{0}, s_{k} \stackrel{\tau}{\longrightarrow} s_{k+1}$ and $s_{k} \models \varphi$ for all $k \in \omega$.

We denote by $\Phi_{j b}^{\widehat{\Delta}}$ the set of formulas generated by the grammar in (1) with $\Delta \varphi$ replaced by $\widehat{\Delta} \varphi$.

Note that the modality $\Delta$ can be expressed in terms of $\widehat{\Delta}$ :

$$
\Delta \varphi \Leftrightarrow \top \tau \widehat{\Delta} \varphi
$$




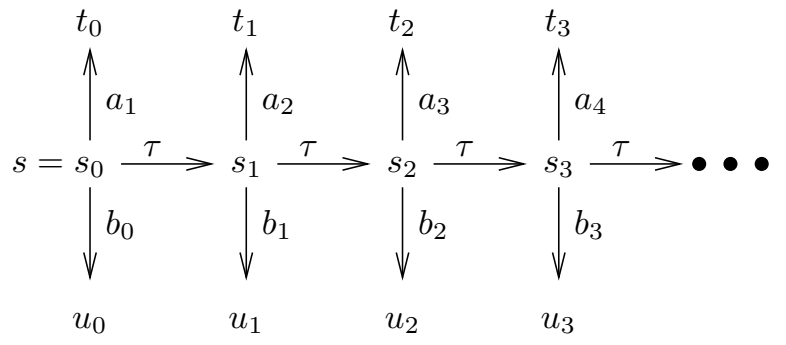

Figure 11. A divergence.

A crucial step in our adaptation of the method of Laroussinie, Pinchinat \& Schnoebelen above consisted of showing that infinite disjunctions in the left argument of $\langle a\rangle$ can be avoided. If infinite disjunctions could also be avoided as an argument of $\widehat{\Delta}$, then a further adaptation of the method would be possible, showing that replacing $\Delta$ by $\widehat{\Delta}$ in the modal logic for $\mathrm{BB}^{\Delta}$ would yield a logic with equal expressivity. However, the following example suggests that infinite disjunctions under $\widehat{\Delta}$ cannot always be avoided.

Example 6.1. Let $a_{1}, a_{2}, a_{3}, \ldots$ and $b_{0}, b_{1}, b_{2}, \ldots$ be infinite sequences of distinct actions and consider the formula

$$
\varphi=\widehat{\Delta}\left(\bigvee_{i=0}^{\infty}\left(\neg\left(\top\left\langle a_{i}\right\rangle \top\right) \wedge\left(\top\left\langle b_{i}\right\rangle \top\right)\right)\right) .
$$

The formula $\varphi$ holds in a state iff there exists an infinite $\tau$-path such that in every state there is an $i \geq 0$ such that the action $b_{i}$ is still possible, whereas the action $a_{i}$ is not. Note that $\varphi$ holds in the state $s$ of the transition system in Figure 11, each of the disjuncts $\neg\left(\top\left\langle a_{i}\right\rangle \top\right) \wedge\left(\top\left\langle b_{i}\right\rangle \top\right)$ holds in precisely one state.

We conjecture that the formula of Example 6.1 is not equivalent to a formula in $\Phi_{j b}^{\Delta}$, and that, hence, replacing $\Delta$ by $\widehat{\Delta}$ in the modal logic for $\mathrm{BB}^{\Delta}$ yields a strictly more expressive logic. We conclude the paper with a proof that the equivalence $\approx^{\widehat{\Delta}} \subseteq S \times S$ induced on states by validity of formulas in $\Phi_{j b}^{\widehat{\Delta}}$, defined by

$$
s \approx^{\widehat{\Delta}} t \quad \text { iff } \quad \forall \varphi \in \Phi_{j b}^{\widehat{\Delta}} . s \models \varphi \Leftrightarrow t \models \varphi
$$

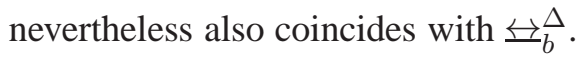

Theorem 6.3. For all states $s$ and $t: s \bigsqcup_{b}^{\Delta} t$ iff $s \approx^{\widehat{\Delta}} t$.

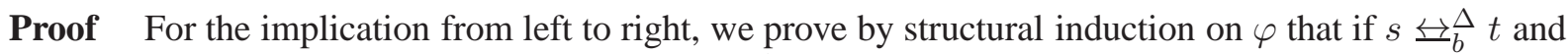
$s \models \varphi$, then $t \models \varphi$. We only treat the case $\varphi=\widehat{\Delta} \psi$, for the cases $\varphi=\neg \psi, \varphi=\bigwedge \Psi$ and $\varphi=\psi a \chi$ are already treated in the proof of Theorem 6.1 So, suppose $\varphi=\widehat{\Delta} \psi$ and $s \models \varphi$. Then there exists an infinite sequence $\left(s_{k}\right)_{k \in \omega}$ of states such that $s=s_{0}, s_{k} \stackrel{\tau}{\longrightarrow} s_{k+1}$ and $s_{k} \models \psi$ for all $k \in \omega$. From 


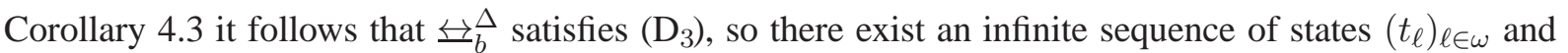

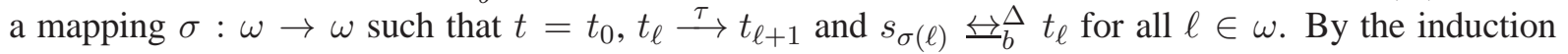
hypothesis $t_{\ell} \models \psi$ for all $\ell \in \omega$, and hence $t \models \varphi$.

To establish the implication from right to left, note that if $s \approx^{\widehat{\Delta}} t$, then, since every formula in $\Phi_{j b}^{\Delta}$ is

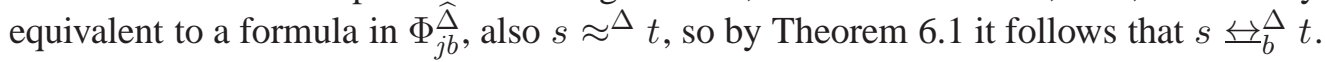

Comment on Definition 2.3 If in Definition 2.3 we had used a notion of equivalence between modal formulas $\varphi$ and $\psi$ that merely requires that $s \models \varphi \Leftrightarrow s \models \psi$ for all states $s$ in the presupposed labelled transition system, rather than quantifying over all labelled transition systems, the resulting concept of equally expressive logics would be much weaker, and the logics $\Phi_{j b}^{\Delta}$ and $\Phi_{j b}^{\widehat{\Delta}}$ would be equally expressive.

In general, let $\sim$ be an equivalence on the set of states $S$, and consider two logics $\mathcal{L}_{1}$ and $\mathcal{L}_{2}$ that both have negation and arbitrary infinite conjunction, and both characterise $\sim$. For every pair of states $s, t \in S$ with $s \not t$ take a formula $\varphi_{s, t}$ from $\mathcal{L}_{1}$ such that $s \models \varphi_{s, t}$ but $t \not \models \varphi_{s, t}$. Then $\chi_{s}=\bigwedge\left\{\varphi_{s, t} \mid t \not s\right\}$ is called a characteristic formula of $s$ : one has $t \models \chi_{s}$ iff $t \sim s$. Now let $\psi$ be a formula from $\mathcal{L}_{2}$. Then $\bigvee\left\{\chi_{s} \mid s \models \psi\right\}$ is equivalent to $\psi$, in the sense that $t \models \psi \Leftrightarrow t \models \bigvee\left\{\chi_{s} \mid s \models \psi\right\}$ for all states $t \in S$. This proves that the two logics are equally expressive.

Similar reasoning using the notion of equivalence from Definition 2.3 would break down, because one cannot take conjunctions of a proper class of formula.

\section{References}

[1] T. Basten (1996): Branching bisimilarity is an equivalence indeed! Information Processing Letters 58(3), pp. $141-147$.

[2] R. De Nicola \& F.W. Vaandrager (1995): Three logics for branching bisimulation. Journal of the ACM 42(2), pp. 458-487.

[3] R. Gerth, R. Kuiper, D. Peled \& W. Penczek (1999): A partial order approach to branching time logic model checking. Information and Computation 150(2), pp. 132-152.

[4] R.J. van Glabbeek (1993): The linear time - branching time spectrum II. In E. Best, editor: Proceedings CONCUR'93, LNCS 715, Springer, pp. 66-81.

[5] R.J. van Glabbeek, B. Luttik \& N. Trčka: Computation tree logic and deadlock detection. Submitted. Available at http://theory.stanford.edu/ rvg/abstracts.html\#73

[6] R.J. van Glabbeek \& W.P. Weijland (1996): Branching time and abstraction in bisimulation semantics. Journal of the ACM 43(3), pp. 555-600.

[7] M. Hennessy \& R. Milner (1985): Algebraic laws for nondeterminism and concurrency. Journal of the ACM 32(1), pp. 137-161.

[8] F. Laroussinie, S. Pinchinat \& Ph. Schnoebelen (1995): Translations between modal logics of reactive systems. Theoretical Computer Science 140(1), pp. 53-71.

[9] S. Nejati (2003): Refinement relations on partial specifications. Master's thesis, University of Toronto. 